Hefte zur Unfallheilkunde

Beihefte zur Zeitschrift ..Der Unfallchirurg“

Herausgegeben von:

J. Rehn, L. Schweiberer und H. Tscherne

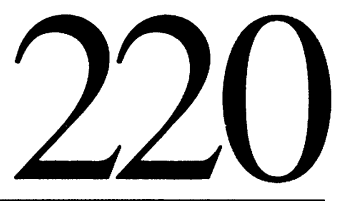




\section{Jahrestagung \\ der Deutschen Gesellschaft \\ für Unfallheilkunde e.V.}

28. November bis 1. Dezember 1990, Berlin

Kongreßthemen: Geschlossene Gelenkverletzungen (ohne Gelenkfrakturen - Muskel-/Sehnentransfer bei Defekt und Fehlheilung an den Gliedmaßen - Röntgendiagnostik am Unfalltag: Effektivität und Effizienz - Polytrauma - Scores: Aussagefähigkeit und Vergleichbarkeit Multiorganversagen-Sepsis - Krankenhaushygiene: Aktuelle Aspekte Qualitätssicherung in der interdisziplinären Akutversorgung des Schwerverletzten - Vorlesungen - Rehabilitation nach Unfällen mit Schädelhirnverletzungen - Freie Vorträge - Forum Experimentelle Unfallchirurgie - Interdisziplinäre Fortbildung - Wissenschaftliche Ausstellung, Posterausstellung - Wissenschaftliche Filme/Video - Schlußveranstaltung

Präsident: A. Pannike Zusammengestellt von K. E. Rehm

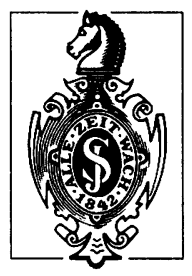

Springer-Verlag Berlin Heidelberg New York London Paris Tokyo Hong Kong Barcelona Budapest 


\section{Inhaltsverzeichnis}

Wissenschaftliches Programm $\ldots \ldots \ldots \ldots \ldots \ldots \ldots \ldots \ldots \ldots \ldots \ldots \ldots \ldots \ldots \ldots \ldots$

Eröffnungssitzung $\ldots \ldots \ldots \ldots \ldots \ldots \ldots \ldots \ldots \ldots \ldots \ldots \ldots \ldots \ldots \ldots \ldots \ldots \ldots \ldots \ldots \ldots \ldots$

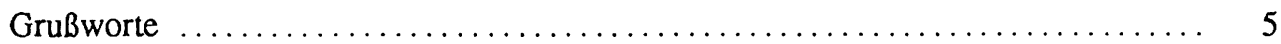

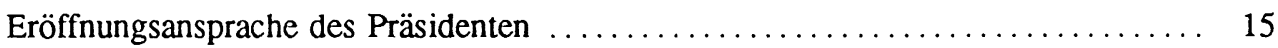

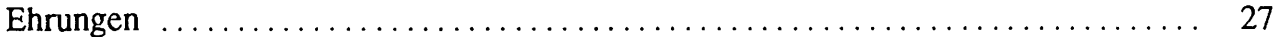

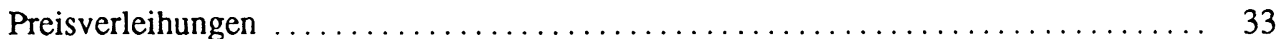

Festvortrag

Ärztliche Kompetenz zwischen medizinischem Fortschritt

und gesundheitspolitischer Verantwortung (H. J. Bochnik) $\ldots \ldots \ldots \ldots \ldots \ldots \ldots . \quad 37$

I. Geschlossene Gelenkverletzungen (ohne Gelenkfrakturen) $\ldots \ldots \ldots \ldots \ldots \ldots$

Überprüfung der Indikation zu konservativer/operativer

Behandlung auf der Basis von Spätergebnissen (> 10 Jahre) $\ldots \ldots \ldots \ldots \ldots \ldots .47$

Die Bedeutung des Spätergebnisses in der Unfallchirurgie

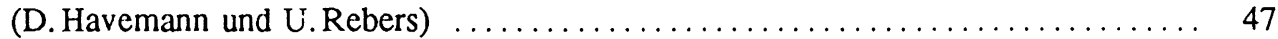

Konservative Behandlung der Schulterluxationen 1969-1980,

Ludwigshafener Ergebnisse (M. N. Magin, H. Winkler, K. Molls, D. Jentschura

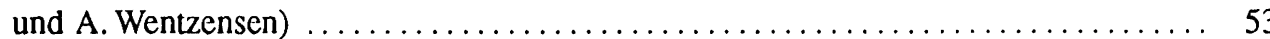

Die modifizierte Operation nach Max Lange in der Behandlung der

posttraumatisch rezidivierenden Schultergelenksluxation. Eine kritische Analyse anhand von Langzeitergebnissen von 18 Jahren bei 70 Patienten

(T.Leonhard, E. Schmidt und M. Schiltenwolf) $\ldots \ldots \ldots \ldots \ldots \ldots \ldots \ldots \ldots \ldots$

Spätergebnisse nach operativer Stabilisation bei posttraumatischer

rezidivierender Schulterluxation (F. Möller, U. von Deimling und D. Doppstadt) ... 56

Spätergebnisse nach Arthrolyse und Arthroplastik

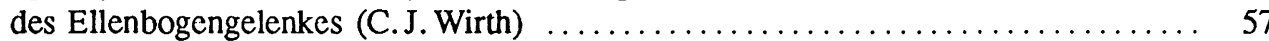

Konservative Behandlung der Ellenbogenverrenkung nach dorsal

(L. Bode und P. Hertel) 
Die traumatische Hüftgelenksverrenkung (B. Niederwieser und Ch. Primavesi) . . . 59

Traumatische Hüftverrenkung (R. Wölfel, W.Link, F. F. Hennig und H. Beck) . . . 60

Spätergebnisse nach traumatischer Kniegelenksluxation

(Ch. Primavesi, F. Genelin, W. Moosmüller und B. Niederwieser)

Spätergebnisse nach operativer Versorgung von Bandrupturen

des Kniegelenkes (F. Genelin, A. Torst, J. Obrist und Ch. Primavesi)

Frische Ruptur des vorderen Kreuzbandes - Resektion der Stümpfe,

Naht oder Plastik (P. Lobenhoffer, M. Blauth und H. Tscherne)

Autologer Kreuzbandersatz mit freiem, knöchern armiertem Patellarsehnendrittel

(K.-A. Riel, H. Weinhart, G. Rübsaamen und P. Bernett) . ..............

Verrenkungen im unteren Sprunggelenk (K. Wenda, J. Degreif,

Th. Sennerich und W. D. v. Issendorff)

Freie Vorträge: I. Geschlossene Gelenkverletzungen $\ldots \ldots \ldots \ldots \ldots \ldots \ldots \ldots$

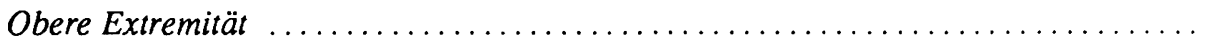

Bietet die Rekonstruktion der Schultereckgelenksprengung mit resorbierbarem Nahtmaterial Vorteile gegenüber der Osteosynthese? Vergleichende Untersuchung der Spätergebnisse 1976-1988 (M. Hahn, K. Neumann und G. Muhr) . . . . . . . . . .

Die Rekonstruktion des Schultereckgelenks.

Vergleich dreier unterschiedlicher Operationsverfahren

(M. Pfahler, A. Krödel und H. J. Refior) $\ldots \ldots \ldots \ldots \ldots \ldots \ldots \ldots \ldots \ldots \ldots$

Instabilität des Schultergelenks: Wert dynamischer Untersuchungsmethoden

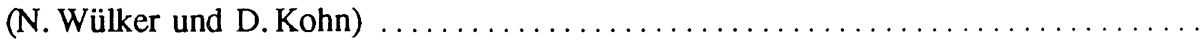

Sonographischer Nachweis der Gelenkstabilität des glenohumeralen Gelenkes

(J.Jerosch, M. Marquardt und M. Schilgen)

Behandlungskonzept für die traumatische Schulterverrenkung

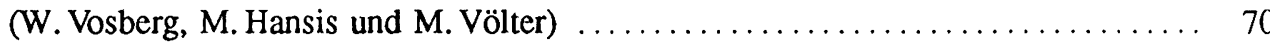

Die hintere Schulter-Subluxation: Differenzierte Diagnostik-Therapie

(J. Brand, A. Ekkernkamp und K. Neumann)

Bankart-Läsionen bei akuter und chronischer vorderer Schulterinstabilität:

Arthroskopische Refixation (N.P. Südkamp, P. Lobenhoffer, N. P. Haas

und $\mathrm{H}$. Tscherne)

Ellenbogenluxation: Differenzierte Therapie-Ergebnisse

(M. Blauth, N. Haas und H. Tscherne)

Ellenbogenluxation - Spätergebnisse

(V. Studtmann, H. Rudolph, und D. Krauss) 
Ellenbogenluxationen ohne/mit Begleitverletzungen des Knochens

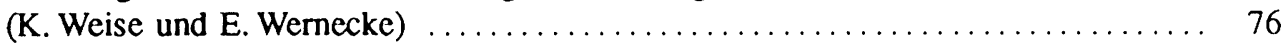

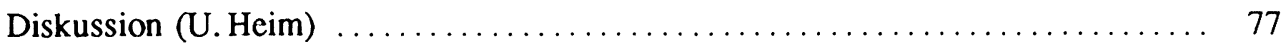

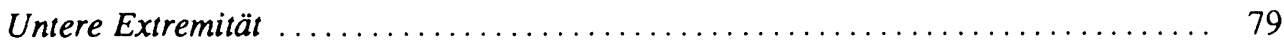

Traumatische Verrenkung des Hüftgelenkes, interdisziplinäre Ergebnisprüfung

(K. E. Dreinhöfer, S. R. Schwarzkopf, M. Prokop, Ch. Ehrenheim und N. Haas) . . . 79

Behandlungskonzept und Spätresultate bei Hüftluxationen

(W. Schlickewei, E. H. Kuner und B. Elsässer) $\ldots \ldots \ldots \ldots \ldots \ldots \ldots \ldots \ldots \ldots \ldots$

Behandlung der geschlossenen Verrenkung des Kniegelenkes

(F. Barnbeck, H. Böhm und G. Hierholzer) $\ldots \ldots \ldots \ldots \ldots \ldots \ldots \ldots \ldots \ldots \ldots \ldots$

Verrenkungen der Sprunggelenke und der Fußwurzel

(H. Zwipp, E. Scola, U. Schlein und D. Riechers) $\ldots \ldots \ldots \ldots \ldots \ldots \ldots \ldots \ldots$

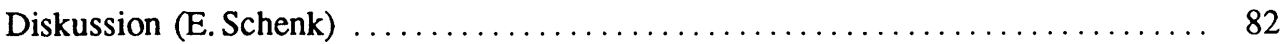

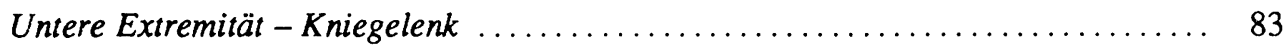

Komplikationen der Kniegelenksarthroskopie - prospektive Studie

(J. Klein, H. Steffens, D. Rixen und Th. Tiling) $\ldots \ldots \ldots \ldots \ldots \ldots \ldots \ldots \ldots \ldots$

Diagnostik und Indikationsstellung beim Hämarthros des Kniegelenkes

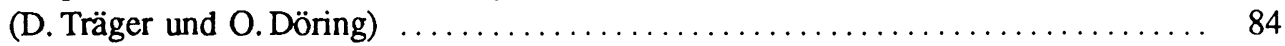

Die Bedeutung der intraarticulären Druckschwankungen für den

chondro-synovialen Stoffwechsel (F. G. Machan, E. Trägenapp und D. Giese) ..... 85

PMN-Elastase und Prokollagen-III-Pcptid in traumatischen Kniegelenksergüssen

(K.-A. Riel, M. Jochum, P. Bernett und H. Fritz) $\ldots \ldots \ldots \ldots \ldots \ldots \ldots \ldots \ldots \ldots$

Sonographische Messung der Instabilität des Kniegelenkes

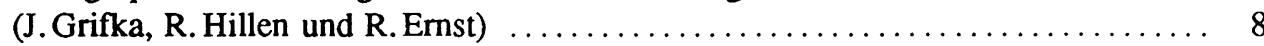

Eine prospektive Untersuchung zur Wertigkeit der diagnostischen Sonographie bei Knieverletzungen (J. Jerosch, M. Schröder und W. H. M. Castro) . ......... 88

Die sonographische Darstellung der Meniscusläsion und ihre Techniken -

Experimentelle Grundlagen am Leichenpräparat

(R. Fenkl, P. Barth und L. Gotzen) $\ldots \ldots \ldots \ldots \ldots \ldots \ldots \ldots \ldots \ldots \ldots \ldots \ldots \ldots \ldots$

10-Jahresergebnisse nach Innenmeniscus-Korbhenkelresektion

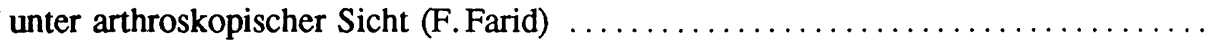

Radiärriß des Außenmeniscus - Eine biomechanische Studie

(D. Kohn, W. Plitz und Th. Muassack) 
Ersetzt die Kernspintomographie die diagnostische Arthroskopie

bei Meniscus- oder Bandverletzungen des Kniegelenkes?

(J. Jerosch, A. Lahm und W. H. M. Castro) $\ldots \ldots \ldots \ldots \ldots \ldots \ldots \ldots \ldots \ldots \ldots$

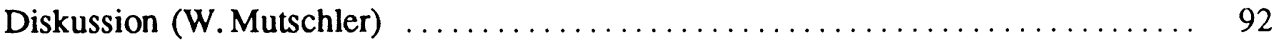

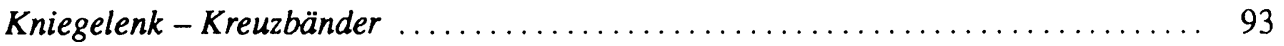

Die Rekonstruktion des vorderen Kreuzbandes - klinisch magnetresonanz-

tomographischer Langzeitverlauf (R. Gradinger, R. Ascherl,

Ch. Kinast, M. Scheyerer, H. Rechl und E. Hipp) $\ldots \ldots \ldots \ldots \ldots \ldots \ldots \ldots \ldots \ldots \ldots \ldots \ldots$

Ersatz des vorderen Kreuzbandes durch den medialen Anteil

des Ligamentum patellae mit schraubenfreier stabiler Verankerung

(P. Hertel, M. Bernard, E. Lais und M. Gomez)

Kreuzbandruptur - Spätergebnisse

(H. Rudolph, V.Studtmann und H.J. Herberhold) $\ldots \ldots \ldots \ldots \ldots \ldots \ldots \ldots \ldots$

Belastbarkeit nach freiem Sehnentansfer: Kreuzbandersatz

mit der Patellarsehne (P. Lobenhoffer, A. Cassim, N. Haas und H. Tscherne) ......

Nachuntersuchungsergebnisse der Ersatzplastik des vorderen

Kreuzbandes mit der distal gestielten Gracilissehne und

Augmentationsplastik mittels PDS-Kordel (Polydioxanon)

(P.-L.Petersen, U. Mommsen und S. Bredendieck) $\ldots \ldots \ldots \ldots \ldots \ldots \ldots \ldots \ldots$

Kreuzbandersatz mit heterologen Bindegewebsstrukturen

(G. Rosbach, L.Zichner und A. Jäger)

Belastbarkeit nach alloplastischem Ersatz im Kniegelenk

(L. Wessel, G. Scheuba und N. Hanhart)

Wesen und Bedeutung der Synovialisreaktionen auf C-Faser-

und Goreteximplantate zum vorderen Kreuzbandersatz

(H. Schweikert, H.-P. Scharf und W.Puhl) ....................... 10

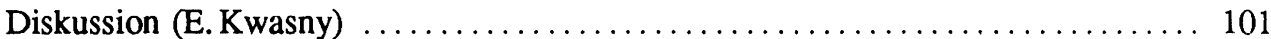

II. Muskel-/Sehnentransfer bei Defekt und Fehlheilung an den Gliedmaßen.

Heutiger Stand - Neubewertung bewährter Verfahren $\ldots \ldots \ldots \ldots \ldots \ldots \ldots \ldots$

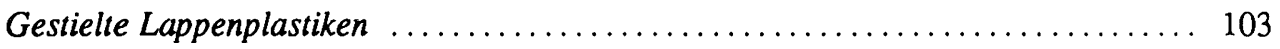

Gestielte Lappenplastiken: Entwicklung und heutiger Stand

am Beispiel der unteren Extremität (H. U. Steinau) $\ldots \ldots \ldots \ldots \ldots \ldots \ldots \ldots \ldots$

Sartorius-Muskellappenplastik bei Weichteildefekten am Becken

(H. Winkler und A. Wentzensen) 
Ist die lokale Muskellappenplastik an den unteren Extremitäten

heute noch sinnvoll? (H. B. Reith, W. Böddecker und W. Kozuscheck) 107

Der lokale Muskeltransfer bei Defekt- und Fehlheilung am Unterschenkel

(U.J.Hesse und K.E. Rehm)

Gestielte Muskellappenplastiken bei offenen Unterschenkelbrüchen

(I. Štraus und J. Princic)

Primäre Muskellappenplastiken zur Defektdeckung bei 1.- und 2.-gradig offenen Unterschenkelfrakturen (A. Bettermann, K. Kunze und C. Schnecker)

Lokale Lappenplastiken am Unterschenkel

(W. Knopp, G. Muhr, M.S. Mackowski und H.U.Steinau)

Regionale fasciocutane, myocutane und Muskellappenplastiken

bei der Behandlung offener Unterschenkelfrakturen

(N. Südkamp, N. Haas, A. Berger und H. Tscherne)

Lokaler Muskeltransfer bei Defekt- und Fehlheilung am Unterschenkel

(J.E. Müller und M. Hansis)

Gestielte Muskellappenplastik am distalen Unterschenkel

(V. Jirecek und N. Ganzoni)

Diskussion (H. Winkler)

Weichteilersatz bei chronischer Osteitis

Ortsständiger Gewebetransfer im Behandlungskonzept der chronischen

posttraumatischen Osteitis an der unteren Extremität

(W. Mutschler und G. Suger)

Transpositionslappen bei posttraumatischer Osteitis und Defekt

(H. Gerngroß und R. Steinmann)

Freie Übertragung von Muskel- und fasciocutanen Lappen zur Behandlung

der chronischen posttraumatischen Osteitis an der unteren Extremität

(M. C. Wüstner, A. K. Hofmann und W. Mutschler)

Weichteildefektdeckung bei chronischer Osteitis

(M. S. Mackowski, W. Knopp, K. Wanner und G. Muhr)

Freie und lokale Muskellappen in der Behandlung von chronischen

Knocheninfektionen (R. Ketterl, H. U.Steinau, B.Stübinger und B. Claudi)

Diskussion (B. Friedrich)

Sekundärer Transfer bei Muskel-/Sehnendefekten: Obere Extremität

Modifizierte Muskelmobilisation nach Debeyre - Indikation, Technik

und Ergebnisse (H. Resch, G. Sperner, K. Golser und H. Thöni) 
Muskel- und Sehnentansfer bei Defekt und Fehlheilung an der oberen Extremität

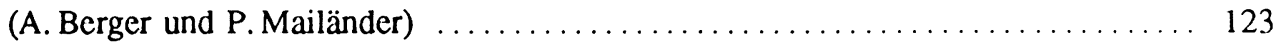

Modifizierte Perthes-Plastik für den Ersatz der Extensoren des Unterarmes (H. Troeger)

Umlagerung des Zeigefingerstreckers zum Ersatz der langen Daumenstreckschne (U. Albers, D. Buck-Gramcko und U. Bültmann) .

Transposition des Extensor indicis zur Rekonstruktion der Extensoren-Sehnen des Daumens und der Langfinger (J. Hoch, G. M.Lösch und M. Schrader)

Extensor-indicis-Plastik zur Wiederherstellung der Streckfähigkeit

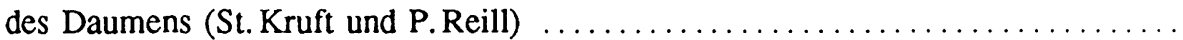

Der Indicis-proprius-Transfer im Vergleich zur freien Sehnentransplantation bei Defekten des Extensor pollicis longus (P. Schaller, B.Landsleitner

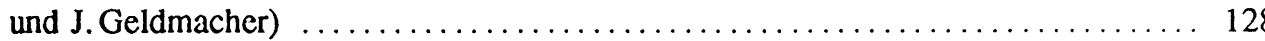

Extensor-indicis-Plastik - Besondere Indikationen (M. Trauner und J. Probst) . . . . 129

Ergebnisse nach Transposition des Extensor indicis zur Wiederherstellung der Daumenstreckung nach Ruptur der Sehne des Extensor pollicis longus (S. Winckler, E. Brug und V.Siebel)

Muskel-Sehnentransfer an der Hand - Technik und Ergebnisse

(H. Towfigh)

Lange Beugesehnentransplantate zur Rekonstruktion veralteter Beugesehnendurchtrennungen. Statische versus dynamische Verbandanordnung (M. Leixnering und W. Hintringer)

Sekundärer Transfer bei Muskel-/Sehnendefekten: Untere Extremität

Sekundäre Wiederherstellung des Kniestreckapparates und glcichzeitige stabile Weichteildeckung durch kombinierte Achillessehnenplastik und M.-gastrocnemius-Lappen (R. Neugebauer)

Die Peronaeus-brevis-Plastik bei großen Defekten der Achillessehne (J.Hassenpflug und W. Blauth)

Korrektureingriff bei Kurzfußsyndrom nach Unterschenkeltrauma

(E. Peterneck, G. Muhr und M. Cordes)

Die modifizierte Elmslie-Tenodese zur Behandlung der Instabilität des unteren Sprunggelenkes - Langzeitergebnisse

(H. Thermann, H.Zwipp und H. Tscherne)

Der Flexor-Extensor-Transfer bei posttraumatischer Zehenfehlstellung (G. Bauer und W. Mutschler) 
Sehnentransfer bei Extremitätentumoren: Erfahrungsbildung

für die Wiederherstellung bei posttraumatischem Defekt und Funktionsverlust

(H. U. Steinau, E. Biemer, J. Schaff und B. Claudi) ................... 139

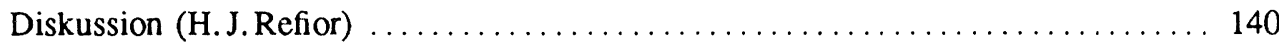

Freie Vorträge: II. Muskel-/Sehnentransfer bei Defekt und Fehlheilung an den Gliedmaßen. Heutiger Stand - Neubewertung bewährter Verfahren ... 141

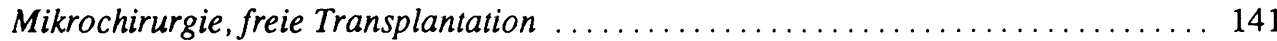

Der tendofasciocutane Dorsalis-pedis-Lappen: Eine Möglichkeit zur

Wiederherstellung der Streckfunktion der Hand bei langstreckigen Sehnendefekten

(S. Eren, P. Hahn, O. Paar und R. Hettich) $\ldots \ldots \ldots \ldots \ldots \ldots \ldots \ldots \ldots \ldots \ldots \ldots \ldots \ldots \ldots$

Differenzierte Indikationsstellung zur mikrochirurgischen Defektdeckung

an der unteren Extremität (J.C. Bruck, R. Büttemeyer und A. Grabosch)

Mikrovasculärer Muskeltransfer bei Defekt- und Fehlheilung an den Extremitäten

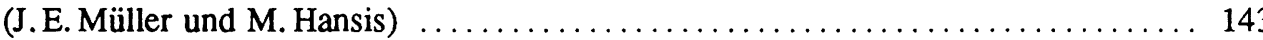

Rekonstruktion großer defektbelasteter Fußsohlenareale mit mikrochirurgischem Transfer (C. Braun, A. Olinger, V. Bühren und M. Bauer)

\section{Röntgendiagnostik am Unfalltag: Effektivität und Effizienz}

Beispiel: Schädel-Hirn-Trauma

Radiologische Diagnostik der Schädelverletzung am Unfalltag:

Einführung in die Problematik (J. Kollath)

Effizienz und Konsequenz der Röntgenuntersuchung beim Schädeltrauma

(J.Richter und E. Wihsgott)

Zur Indikation der ambulanten Röntgenuntersuchung des Schädels

am Unfalltag (Multizenterstudie) (J. Windolf, R. Inglis und A.Pannike)

Schädelhirntrauma: Wert der Röntgenuntersuchung des Schädels in 2 Ebenen

(G. Steinau, F.P.Pfingsten, B. Dreuw und O.Paar)

Fehldiagnostik bei Schädelhirntraumen - forensische Aspekte

(H. Bratze und R.Penning)

Verbesserung von Effektivität und Effizienz der Röntgenuntersuchung des Schädels durch Unfallanamnese und klinischen Befund?

(E. Scheller, A. Meißner und R. Rahmanzadeh)

Effektivität von Notfall-CT und konventionellen Röntgenaufnahmen des Schädels beim Schädel-Hirn-Trauma (SHT) (S. A. Beyer-Enke, F. Bäumer, S.Zrinzo, H.-W.Stedtfeld, E.Zeitler und A. Settele) 
Das Schädel-Hirn-Trauma - Effektivität und Effizienz der bildgebenden Verfahren am Unfalltag (Chr. Reith und H. B. Reith)

Radiologische Akutdiagnostik beim Schädel-Hirn-Trauma

Frühzeitige CT-Diagnostik bei frontobasalen Schädelhirnverletzungen

(P. Knöringer)

Kranielle Computertomographie in der Akutphase des Schädel-Hirn-Traumas

(R. Weinstabl, H. Schurawitzki, F. Kutscha-Lissberg, O. Kwasny und W. Scharf) . . 154

Wertigkeit der Ultra-Low Field-Magnetresonanz (ULF-MR) - Untersuchung beim akuten Schädel-Hirn-Trauma (A. Janousek, G. Rappold und G.Siakos) ..... 156

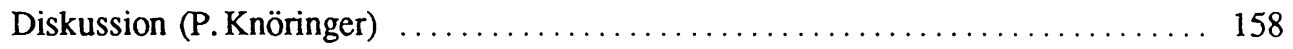

Freie Vorträge:

III. Röntgendiagnostik am Unfalltag: Effektivität und Effizienz 161

Allgemeine und Radiologische Diagnostik: Schädelhirntrauma, Polytrauma

Gefäßverletzung

Versorgung des schweren gedeckten SHT im Krankenhaus

Paul-Gerhard-Stift Wittenberg (J.Dörfel)

Schädelverletzung beim Polytrauma: Anlaß zur Verlegung in ein

Schwerpunktkrankenhaus - Fehlbeurteilung lebensbedrohender Zusatzverletzungen

(M. Varney, H. Becker und H.-D. Röher

Schnelle Diagnostik polytraumatisierter Patienten durch digitale Röntgentechnik

(D. Büscher, J. Büsselberg, U. Flesch, P. Hertel und H. Witt)

Verbesserung der radiologischen Diagnostik des Polytraumas

durch digitale Lumineszenzradiographie

(Th. Hilbertz, H. Berger, T. Mittelmeier und G.Lob)

Moderne bildgebende Diagnostik der traumatischen Carotisdissektion

(W. Crone-Münzebrock, U. Grzyska, P.-P. Spielmann und N. M. Meenen)

Diagnostik vasculärer Begleitverletzung beim Polytrauma -

Katheterembolisation in der Akutsituation

(H. Berger, T. Hilbertz, H. Dienemann und G.Lob) 166

Röntgendiagnostik am Unfalltag: Fraktur und Gefäßverletzung -

Wann ist eine Notfallangiographie indiziert?

(W. Schlickewei, E. H. Kuner, G. Spillner und B. Goetze)

Polytrauma: Primär nicht erkannte Verletzungen des Stütz- und Bewegungsapparates (G. Metak, Ch. Dannöhl und W. Heitland) 
Szintigraphische Kontrolle der primären Röntgendiagnostik bei Polytraumatisierten (M. Runkel, H. Steinert, W. Röder und $\mathrm{K}$. Wenda)

Röntgendiagnostik am Unfalltag: Halswirbelsäulenverletzungen

bei Schädel-Hirn-Trauma (W. Mutschler, M. Arand und P. Schnarkowsky)

Ist die konventionelle Röntgendiagnostik zur Beurteilung von HWS-Verletzungen beim Mehrfachverletzten ausreichend?

(H. Hertlein, S. Piltz, A. Stäbler, H. Berger und G. Lob)

Die CT-Untersuchung bei traumatischer Schädigung der Halswirbelsäule -

Eine präoperative Notwendigkeit? (M. Hahn, O.Russe, U. Bötel und G. Muhr)

Röntgendiagnostik des Wirbelsäulentraumas am Unfalltag:

Indikation und Effektivität tomographischer Verfahren

(Th. Heuchemer, H. Waidelich, J. Häberle und G. Bauer)

Diagnostik traumatischer Frakturen der BWS/LWS -

Wertigkeit verschiedener bildgebender Verfahren

(M. Schax, K. M. Stürmer, K. Koeser und M. Serdarevic)

Intraoperative Myelographie, prä- und postoperatives CT.

Vergleich der Wertigkeit bei der Sofort- oder Frühversorgung instabiler Brüche

der BWS und LWS (O.Russe, U. Bötel und A. Biebach)

Eine klinische Klassifikation von thoraco-lumbalen Wirbelsäulenverletzungen Eine einfache und eindeutige Einteilung mit therapeutischer Implikation anhand konventioneller Röntgendiagnostik (R. Sambale, P. Metz und V.Echtermeyer)

Diskussion (B. Wimmer)

Radiologische Diagnostik beim Thoraxtrauma

Ist die Aussagekraft der konventionellen Thorax-Aufnahme beim Polytrauma ausreichend? - Indikation und Wertigkeit einer weiterführenden CT-Diagnostik (J.H. Langkowski, D. Grossner, K. H.Jungbluth und E. Bücheler)

Radiologische Akutdiagnostik des Thoraxtraumas am Unfalltag Ist die Thoraxübersichtsaufnahme ausreichend?

(J. Windolf, S. Gottschalk, R. Inglis und A.Pannike)

Fehlbeurteilung einer Thoraxverletzung bei der Primärdiagnostik bei Polytraumatisierten (L. Rudig, W.Röder, J. Ahlers und P. Grebe)

Thorax-Computertomographie am Unfalltag - Nutzen und Risiko (C. Sangmeister, E. M. Walthers und M. Sangmeister) 
Thorax-Computertomographic am Unfalltag - Nutzen und Risiko

(C. Sangmeister, E. M. Walthers und M. Sangmeister)

Radiologische Strategie bei traumatischen Rupturen der

thorakalen Aorta Abschnitt III (Th. Hilbertz, H. Berger, T. Mittelmeier

H. Dienemann und G. Lob)

Diagnostische Wertigkeit der Sonographie im Vergleich zur

Röntgenuntersuchung beim Thoraxtrauma (M. Walz und G. Muhr) $\ldots \ldots \ldots \ldots \ldots 183$

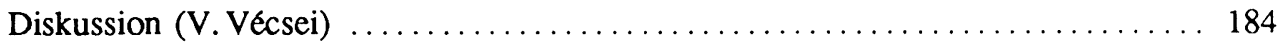

Allgemeine und spezielle radiologische Diagnostik: Obere Extremität ......... 184

Nichterkennung der hinteren Schulterluxation bei der Röntgeneinstellung

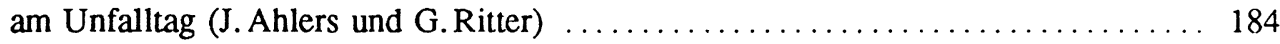

Zusatzinformationen von Kontrastmittel-CT und MR-Tomographie

bei Schulterverletzungen (K. Wenda, K. F. Kreitner, J. Grimm und J. Ahlers) . . . 185

Fettpolsterzeichen und Supinatorfettlinie als indirekte Verletzungsszeichen

am Ellenbogengelenk (S. W. Dihlmann, N. M. Meenen und K. H. Jungbluth) . . . . 186

Radiologische Diagnostik der Monteggiaverletzung am Unfalltag

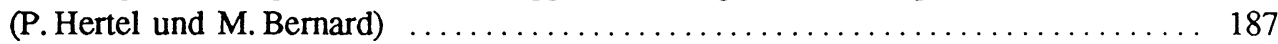

Messung des Knochenmineralgehaltes bei konservativ versorgten

Unterarmfrakturen (J. Frohn, J. Jeibmann, J. M. Rueger R. Inglis, G. Hör

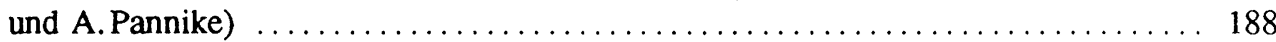

Die frische Scaphoidfraktur - Problematik des radiologischen Nachweises

(Th. Kreusser, M. Nägele, E. Euler und K. Wilhelm) .

Diagnostik der Scaphoidfraktur (E.J.R. van Beek, M. M. M. Tiel-van Buul,

A. H. Broekhuizen E. L.F. B. Raaymakers und A. J. Bakker)

Digitale Luminescenzradiographie zur primären Diagnostik

von Handwurzelverletzungen (H. J. Kock, B. Buddenbrock, R.-D. Müller,

G. Schmidt und M. Voss)

Radiologische Grundlagen der Diagnostik frischer Fingerverletzungen

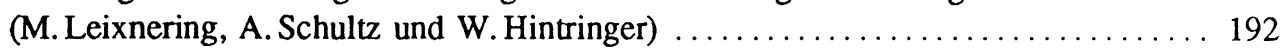

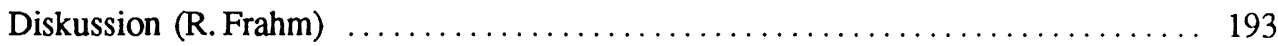

Allgemeine und spezielle radiologische Diagnostik: Untere Extremität . . . . . . . . 194

Vorteile des Computertomogramms gegenüber der konventionellen Röntgentechnik

bei der Beckenringverletzung (W.Röder, K. Wenda, W.-D. v. Issendorff

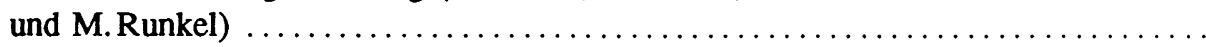

Diagnoseverzögerung bei Hüftgelenksverletzungen - Ursachen und Prophylaxe

(W. Kurock und K. Wenda) ................................... 
MR-Tomographie nach Hüftgelenkstraumen

(K. Wenda, G. Ritter, F. F. Kreitner und J. Ahlers)

Die kontrastmittelunterstützte Magnetresonanztomographie

zur Kontrolle der Hüftkopfdurchblutung nach Schenkelhalsfraktur

(M. Mauz, H. H. Schauwecker, M. Langer und Ph. Lang)

Diagnostik der frischen Ruptur des vorderen Kreuzbandes durch CT

(K. Höcker, N. Schwarz, E. Tipold und W.Zechner)

Wert der Röntgendiagnostik nach Supinationstraumen des

oberen Sprunggelenkes und Fuß (J.J. A. M. van Raay, Chr. van der Werken

und A. V.C.M.Zeegers)

Zusatzinformationen von MR-Tomographie und Szintigramm

bei Talusfrakturen (K. Wenda, L. Rudig, T. Sennerich und W. Kurock)

IV. Polytrauma-Scores: Aussagefähigkeit und Vergleichbarkeit

Die Entwicklung von Score-Systemen (J. A. Sturm)

Trauma Score: Heutiger Stand (H. R. Champion)

(Manuskript nicht eingegangen)

Der Hannoversche Polytraumaschlüssel

(H.-J. Oestern, K. Kabus und C.Neumann)

Glasgow Coma Scale (M. Brock)

(Manuskript nicht eingegangen)

Scores für posttraumatischen Verlauf, Multiorganversagen und Sepsis

(M. L. Nerlich) (Manuskript nicht eingegangen)

Freie Vorträge:

IV. Polytrauma-Scores: Aussagefähigkeit und Vergleichbarkeit

Beurteilung der Verletzungsschwere und Vergleichbarkeit der Scores

Mathematische Voraussetzungen zum Vergleich der Aussagefähigkeit

verschiedener Polytrauma-Scores (R. Friedel, E. Markgraf und J. Schwarz)

Prüfung der ISS- und PTS-Aussagen am eigenen Krankengut

(U. Obertacke, U. Am Orde, F. Rumler und Th. Joka) $\ldots \ldots \ldots \ldots \ldots \ldots \ldots \ldots . \ldots 218$

Einordnung der Schwerverletzten mit AIS- und ISS-System (Z.Záborszky) . . . . 219

Vergleich „europäischer" und ,amerikanischer" Polytrauma-Scores

(T. Braunsteiner, M. Brix, J. Látal und P. Šimko) . .................. 220

Einfluß des Untersuchers auf ISS- und PTS-Scores

(Ch. Waydhas, D. Nast-Kolb und L. Schweiberer) 
Zur Vergleichbarkeit dynamischer Polytraumascores

(R. Inglis, J. Windolf, T. Jünger und A.Pannike) $\ldots \ldots \ldots \ldots \ldots \ldots \ldots \ldots \ldots 22$

Diskussion (J.Poigenfürst)

Scores: Triage, Prognostische Beurteilung, Verlaufsbeurteilung

Welche Polytrauma-Scores sind im Notarztdienst einsetzbar?

(J.Schmidt, K. Konzel und B. Dillmann)

Validität, Reliabilität und Vorhersagewert von Trauma-Scores (TS),

Injury-Severity-Score (ISS) und TRISS-Auswertung von 2074 Trauma-Patienten

1987 im Kölner Rettungsdienst (M. Schweins, B. Bouillon, Th. Tiling und H. Troidl) 225

Klassifikation der Verletzungsschwere anhand des Traumaindex (TI)

von Schreinlechner und Eber (G. Rappold und U.-P. Schreinlechner)

Traumaindex nach Schreinlechner und Injury-Severity-Score -

Vergleich hinsichtlich der Mortalitätsvoraussage

(E. Foltin, F. Helml, C. Rodemund und H. Haller)

Erlauben Polytrauma-Scores prognostische Aussagen zum Langzeitverlauf

nach Polytrauma? (R. Kasperk, O.Paar und S. Eren)

Die prognostische Aussagekraft biochemischer Parameter im Vergleich zum ISS

und PTS beim Polytrauma (D. Nast-Kolb, Ch. Waydhas, M. Jochum

und L. Schweiberer)

Bewertung von Vorerkrankungen und Inhalationstrauma für die

prognostische Sicherheit von Aufnahme-Scores bei Schwerverbrannten

(G. Germann, T. Kuipers und W.Perbix)

Retrospektiv vergleichende Analyse von PTS, TS, TIK, TIS und ISS

hinsichtlich der prognostischen Wertigkeit der Aussage

(A. Stockinger, K.-P. Benedetto und E. Beck)

Beurteilung und Wertung verschiedener Polytrauma-Scores

anhand der Verläufe von 268 polytraumatisierten Patienten

(H. B. Reith, Ch. Reith, W. Böddecker und W. Kozuscheck)

Vergleichbarkeit von Kollektiven polytraumatisierter Patienten:

Trauma-Scores versus personalcomputergesteuerte „One-Line“-Erfassung

des Krankheitsverlaufs (J. Windolf, R. Inglis und A. Pannike)

Der Heidelberger Wachstation-Score (HDWS): Klassifizierungssystem

zur Verlaufsdokumentation und Prognosestellung bei polytraumatisierten Patienten

(T. Foitzik, I. Göhring, M. Betzler und W. Friedl) 


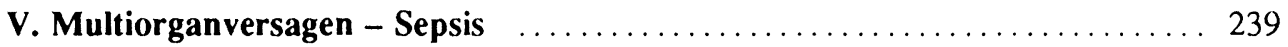

Aktuelle Aspekte - Teil I .................................... 239

Multiorganversagen und Sepsis nach Polytrauma (O. Trentz)

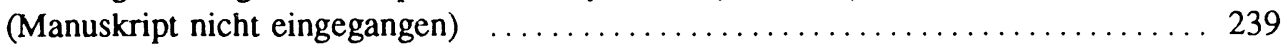

Pathophysiologie des septischen Geschehens nach Trauma (G. Schlag)

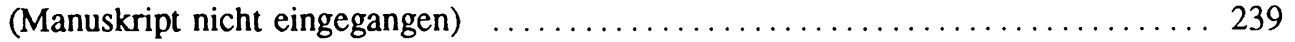

Pathophysiologie der inflammatorischen Akutphasenreaktion nach Trauma

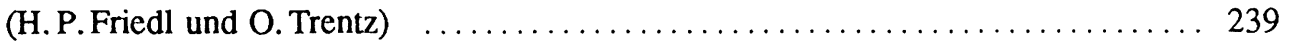

Neue Erkenntnisse zur Pathogenese des cellulären Immunitätsdefektes -

Multiorganversagen und Sepsis (E. Faist)

(Manuskript nicht eingegangen)

Wundinfektion - Systemische Sepsis - Multiorganversagen (M. L. Nerlich)

(Manuskript nicht eingegangen)

Incidenz, Verlauf und klinische Interventionsmöglichkeiten

bei septischen Komplikationen nach Polytrauma (K. H. Duswald)

(Manuskript nicht eingegangen)

Die Bedeutung alveolärer Reaktionen für die Entstehung, Erkennung

und Vorhersage des Multiorganversagens (Th. Joka)

Aktuelle Aspekte - Teil II _.................................... 254

Inhalationstrauma - Sepsis - Multiorganversagen

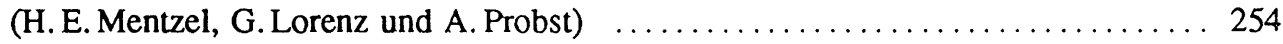

Trauma-induzierte Mucosa-Dysfunktion: Sepsis-Prävention durch nicht-selektive Darmdekontamination und früh-enterale Ernährung (A. Ekkernkamp, J. Brand, G. Möllenhoff und G. Muhr) $\ldots \ldots \ldots \ldots \ldots \ldots \ldots 254$

Das Weichteiltrauma und sein Einfluß auf die unspezifische Immunabwehr (A. Seekamp, A. Dwenger, G. Regel, G. Schweitzer und J. A. Sturm) _........ 255

Einfluß der Verletzungsschwere auf die Keimbesiedlung in der Folgephase (J. Sauer, R. Inglis, G. Klein, J. Windolf, P. Konold und A. Pannike)

Prädiktive Wertigkeit von Plasmaendotoxin, C3a, Leukocytenzahl und Elastase in der Frühphase der Sepsis hinsichtlich des Überlebens (E. Klar, M. Kieser, G.Zilow, K. P. Becker, H. Buhr und Ch. Herfarth) $\ldots \ldots \ldots \ldots \ldots \ldots \ldots \ldots \ldots$

Können polyvalente Immunglobulinpräparationen das Multiorganversagen bei der Sepsis beeinflussen? Experimentelle und klinische Ergebnisse (D. Nitsche, H. Groeper und H. Hamelmann) 
Freie Vorträge: V. Multiorganversagen - Sepsis $\ldots \ldots \ldots \ldots \ldots \ldots \ldots \ldots \ldots$

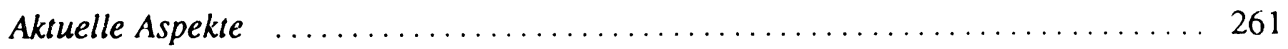

Der Verlauf von Elastase, Neopterin und TNF-Alpha im Serum

polytraumatisierter Patienten (A.F. Hammerle, P. Krafft, A. O. Wagner,

R. Jaskulka, J. Winternitz und P. M. Winter)

Aussagefähigkeit von Serum-Neopterinspiegeln bei Brandverletzten

im Rahmen von Sepsis und Multiorganversagen (A. Grabosch) $\ldots \ldots \ldots \ldots \ldots 262$

Organversagen und Sepsis nach Polytrauma (G. L. Tüchy, M. Fuchs, E. Tüchy

und O.Kwasny)

Verlaufsmessungen der alveolären Proteindurchlässigkeit nach Polytrauma durch serielle bronchoalveoläre Lavage (U. Obertacke, Th. Joka, E. Kreuzfelder und K.P.Schmit-Neuerburg)

Stress-Cholecystitis bei polytraumatisierten Patienten:

Ergebnisse einer prospektiven sonographischen Studie

(J.Raunest, K.P. Thon, M. Imhof und Ch. Ohmann)

Die Bedeutung abdomineller und pelviner Verletzungen für die Incidenz

des Multiorganversagens nach Polytrauma (A.Seekamp, G. Regel, U. Bosch

und J. A. Sturm)

Vergleichende Statistik zur Letalität langzeitbeatmeter polytraumatisierter

Patienten (F. Rumler, U. Am Orde, U. Obertacke und Th. Joka)

Zusammenfassung der Diskussion - Sitzung zum Thema:

Multiorganversagen/Sepsis: Aktuelle Aspekte

VI. Krankenhaushygiene: Aktuelle Aspekte

Infektionsprophylaxe im Operationstrakt

Bauliche Voraussetzungen: Schleusen, Ein-/Ausleitung, Ver-/Entsorgung, Belüftung

(G. Hierholzer und S. Hierholzer)

Infektionsprophylaxe im Operationstrakt - Organisatorische Voraussetzungen

(H. Rudolph)

Desinfektionsverfahren: Hände, Haut (E. Beck)

Sterilisationsverfahren (P. Heeg)

Diskussion (H. Rudolph) 
Aktuelle Aspekte der Krankenhaushygiene. Stellungnahme des Chirurgen und Beratenden Arztes eines Landesverbandes der gewerblichen

Berufsgenossenschaften (J. Probst)

Trennung der Operationsbereiche - ein Anachronismus?

Stellungnahme aus der Sicht eines OP-Pflegers (M. Hilbert) 300

Freie Vorträge: VI. Krankenhaushygiene: Aktuelle Aspekte $\ldots \ldots \ldots \ldots \ldots 305$

Nutzen der Operations-Incisionsfolie - Perioperative, quantitative

und qualitative Keimanalyse (B.-D. Katthagen, P. Aeckerle und H. Mittelmeier) _. 305

Kontinuierliche bakteriologische Kontrolle in der Unfallchinurgie -

Ergebnisse einer prosepktiven 9-Jahres-Studie (M. Hansis) $\ldots \ldots \ldots \ldots \ldots \ldots \ldots 6$

Verfolgung bakterieller Kontaminationswege im hochaseptischen Operationssaal (Reinraumkabine) (H. Freick, B. Friebe und T. Lehners) _............... 307

Staphylokokken - Hospitalismusgefahr in der operativen Orthopädie?

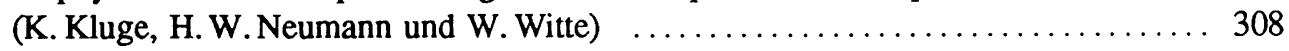

ASA-Antibiogramm-Struktur-Analyse - eine Methode zur

EDV-gestützten Erkennung nosokomialer Infektionen auf Intensivstationen

(H. M. Seipp, H. Knaepler und L. Gotzen) f......................... 309

Hygienestandard bei der Behandlung Brandverletzter

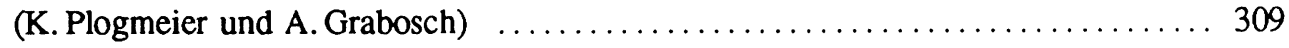

VII. Qualitätssicherung in der interdisziplinären Akutversorgung des Schwerverletzten

Die interdisziplinäre Behandlung des Polytraumatisierten:

Möglichkeiten und Grenzen (J. L. Hughes, Jr.) $\ldots \ldots \ldots \ldots \ldots \ldots \ldots \ldots \ldots \ldots \ldots \ldots \ldots$

Fachinternistische Untersuchung und interdisziplinäres Konsil

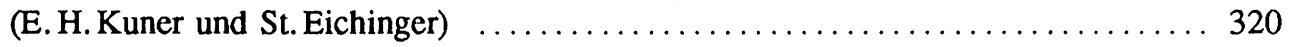

Fachneurologische Untersuchung und interdisziplinäres Konsil (H. Kuderna)

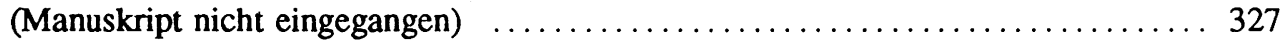

Gemeinsame Akutversorgung mit dem Anaesthesisten (J. A. Sturm) $\ldots \ldots \ldots \ldots, 327$

Qualitätssicherung in Wissenschaft und Klinik $\ldots \ldots \ldots \ldots \ldots \ldots \ldots \ldots \ldots \ldots \ldots \ldots$

Klassifikation als Grundlage der Evaluation: Dargestellt am Beispiel der Frakturklassifikation (M.E. Müller)

(Manuskript nicht eingegangen) 
XXII

Qualitätssicherung und Sorgfaltspflicht in der ärztlichen Begutachtung von Verletzungsfolgen (G. Hierholzer und E.Ludolph)

Qualitätssicherung im Krankenhaus

Qualitätssicherung im Krankenhaus - Rechtsgrundlagen und Problemanalyse

(F. Beske)

Das Krankenblatt - Anmerkungen zur ärztlichen und juristischen Problematik

(Th. Köhler und K. H. Müller)

Problemorientierte Datenerfassung als Grundlage der Qualitätssicherung

in der Unfallchirurgie (Ch. Veit, A. Tecklenburg und F. Hennig)

KIDS, ein EDV-System zur Verarbeitung von Patientendaten in der

Unfallchirurgie (M. Fleck und H.-J. Oestern)

Fallklassifikation als Voraussetzung der Qualitätssicherung: Überprüfung der

Patienten-Management-Categories (PMC) bei chirurgischen Patienten

(H. Bauer und G. Neubauer)

Aussagen einer Leistungserfassung in der Unfallchirurgie für die Organisation von Leistungsstufen (K. Welz)

Implementierung kliniksspezifischer Basisdokumentationssysteme in der Unfallchirurgie (M. Schnabel, M. Künneke, R. Schlenzka, H. Knaepler und L. Gotzen)

Qualitätssicherung durch prospektive Datenerfassung: Modell A. K. St. Georg

(J. Grüber, C. Mella-Schmidt und Ch. Eggers

Computergestützte Dokumentation und Qualitätskontrolle in der Abteilung

für Traumatologie des Bezirkskrankenhauses Suhl (F. Recknagel)

EDV-Einsatz in einer zentralen Operationsabteilung

(R. Salm, P. Münst und F. Heinemann)

Berechnung der individuellen Behandlungskosten bei Patienten auf der Intensivstation durch Gewichtung? - Der Frankfurt-Weighted-Cost-Score (FWCS)

(R. Inglis, J. Windolf und A.Pannike)

Diskussion (K. Welz)

Qualitätssicherung im Krankenhaus: Befunddokumentation - Evaluation 350

Einordnung und Ergebnisprüfung bei der Behandlung Schwerstverletzter -

Ein neuer methodischer Ansatz (E. Reichle, Ch. Eggers und D. Wolter)

EDV-unterstützte Patientendokumentation - Erfahrungsbericht über 3000 unfallchirurgische Patienten (J. Hettfleisch, H. Schöttle, F. Herrmann und W. Beck) 
Qualitätssicherung bei der medialen Schenkelhalsfraktur (O. Scheibe) 353

Explorative Datenanalyse zur Beurteilung der Schraubenosteosynthese bei Schenkelhalsfrakturen (G. Helbing und U.Schmid) $\ldots \ldots \ldots \ldots \ldots \ldots \ldots \ldots 3$

CAD-unterstützte Planung von Osteotomien an der unteren Extremität (F. Ullrich und F. Schauwecker)

Dokumentation arthroskopischer Befunde beim degenerativen Meniscusschaden

(P.D.Platzek, Th. Köhler und K. H. Müller)

Wert der Röntgenuntersuchung bei ligamentären Verletzungen des oberen Sprunggelenkes (C. Wendler, R. Inglis, J. Windolf, D. Liermann, J. Kollath und A.Pannike)

Das Patienten-Monitoring-System: PAMOS (W.Schneider und K.-W. Hartmann) . . 358

Datenbank zur Qualitätssicherung und Statistik bei der primären und sekundären operativen Behandlung handverletzter Patienten (M. Schrader und G. M.Lösch) . . 359

Computerunterstützte Datenerfassung und Analyse von septischen Komplikationen in der Traumatologie (M. Leixnering, Th. Öhner und M. Ben Mokhtar)

VIII. Vorlesungen 361

A. Verlust und Wiedererlangung des „Körperbildes“ nach peripheren Verletzungen (H. W. Delank)

B. Ersatzoperationen bei irreparablen Verletzungen peripherer motorischer Nerven an der oberen Extremität (D. Buck-Gramcko)

(Kein Manuskript)

Freie Vorträge zu Vorlesung B

Obere Extremität

Muskelersatzplastiken bei posttraumatischer Läsion der oberen Antcile

des Plexus brachialis (M. Wiedemann, A. Rüter und A. Narakas)

Ersatzoperationen bei irreparablen Verletzungen des Plexus brachialis

(A. Berger, H.-J. Bargmann und A. Feichter)

Muskel-Sehnentransfer bei unvollständiger Plexusrekonstruktion

(R. Henke)

M. biceps und M.triceps als Kraftträger zur Wiederherstellung der Beuge- und Streckfunktion der Hand bei komplettem Muskelverlust

(S. Eren und R. Hettich)

Lokale und freie Muskelersatzplastiken als optimierende Maßnahme nach Rekonstruktion peripherer Nerven an der oberen Extremität

(H.-J. Bargmann, A. Berger, E. Schaller und P. Mailänder) 
Die Stcindler-Plastik bei Lähmung der Ellenbeuger (A. K. Martini) 380

Biceps-Ersatzoperationen durch Transposition des M. latissimus dorsi

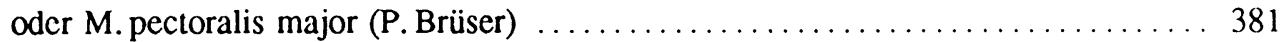

Die Wiederherstellung der Pronation des Unterarmes (A. K. Martini) . . . . .... 381

Mehrsehnenplastik bei Nervus radialis-Paresen: Indiaktion und Ergebnisse

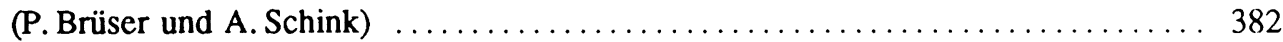

Motorische Ersatzoperation bei irreversibler Radialislähmung (M.Maeß) . ..... 383

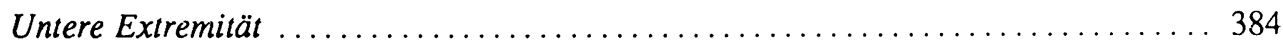

Tibialis posterior-Transfer nach schwerer Knieverletzung und Zerreißung des N. peronaeus (G. Berentey und G. Béres) $\ldots \ldots \ldots \ldots \ldots \ldots \ldots$

Der Tibialis posterior-Transfer nach Kompartment-Syndrom oder Peronaeusparese

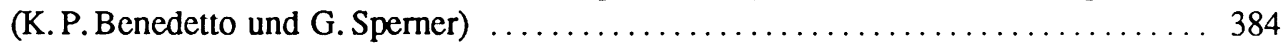

Tibialis posterior-Transfer bei N. peronaeus-Lähmung (H.-W. Ulrich und W. Blauth) 386

Der M. tibialis posterior-Transfer als Ersatzoperation bei N. peronaeus-Läsionen und posttraumatischen Läsionen (J.Schweitzer und F. Shahidi)

Muskeltransposition bei inkompletter und kompletter Peroneusparese Technik - Ergebnisse (A. Georgoulis und P. Hertel) $\ldots \ldots \ldots \ldots \ldots \ldots \ldots \ldots \ldots$

Tibialis posterior-Transfer zur Kompensation des ausgefallenen N. fibularis

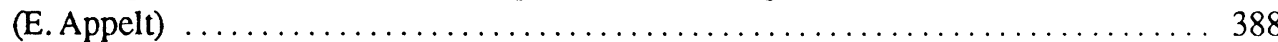

Postoperative Ergebnisse nach Peronaeus-Ersatzplastik

(J.Heisel, T. Siebel, E. Schmitt und H.J. Hesselschwerdt) . . . . . . . . . . . . . 389

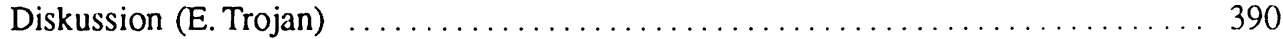

C. Nervenkompressionssyndrome der Gliedmaßen (H. Millesi und D. Eberhard) . . . 391

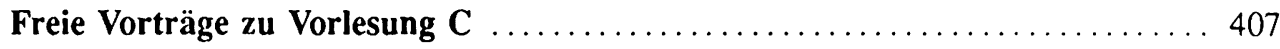

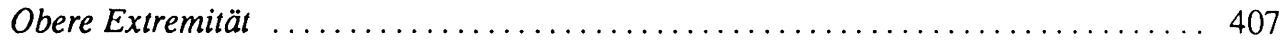

Wertigkeit der Mikroendoneurolyse bei der Kompressionsneuropathie eine tierexperimentelle Sîdie (O. Sölch, E. Markgraf und J.Schwarz) . . . . . . . 407

Dekompression der Nervenengstellen und offene Wundbehandlung bei schwerer Brustwand- und Armphlegmone (A. Obiltschnig und D. Szolar) .... 408

Ulnaris-Rinnensyndrom als Spätfolge von Frakturen des

Epicondylus ulnaris humeri (Th. Sennerich, W. Röder, W. Kurock und V.Karnosky) 
Chirurgisches Vorgehen bei der Neuropathie des N. ulnaris im Bereich

des Ellenbogengelenkes (P.Graf, A.-M. Feller, H.U.Steinau und E. Biemer)

Ergebnisse nach Neurolyse und Verlagerung des N.ulnaris

am Ellenbogen (P. Ansorg und Th. Wolfram)

Carpaltunnelsyndrom als Komplikation der distalen Radiusfraktur

(W. Röder, W.Nix, Th. Sennerich und S.Fischer)

Posttraumatische Kompressionssyndrome des N. medianus im Bereich

des Handgelenkes (R. Henke) .

Kompressionssyndrom des $\mathrm{N}$. interosseus anterior

(A. K. Hofmann und M. C. Wüstner)

Diskussion (Chr. Walke)

IX. Rehabilitation nach Unfällen mit Schädelhirnverletzungen

ZNS: Kuratorium für Unfallverletzte mit Schäden

des Zentralnervensystems e.V.

Ziele und Aufgaben des Kuratoriums ZNS (Hannelore Kohl)

Frührehabilitation Schädelhirnverletzter in der Neurochirurgischen Klinik

(M. R. Gaab)

(Manuskript nicht eingegangen)

Die Frührehabilitation bei Schädel-Hirn-Verletzungen (W. Gobiet)

Zuwendungs- und Förderpraxis des Kuratoriums ZNS -

Zuwendungen durch das Kuratorium ZNS (B.Bom)

Zuwendungs- und Förderpraxis des Kuratoriums ZNS -

Vermittlungsstelle für Rehabilitationsplätze (R. Wiechers)

X. Freie Vorträge

Handchirurgie

Arthroskopische Abklärung unklarer Handgelenksbeschwerden

(T.Pomsel und A. Ahmadi)

Wann ist die scapholunäre Dissoziation eine Gelenkverletzung?

(J. Degreif, G. Ritter, K. Wenda und Th. Sennerich)

Carpale Instabilitäten (E. Euler, Th. Kreusser und K. Wilhelm)

Daumensattelgelenkverrenkungen - Behandlung und

Nachuntersuchungsergebnisse (W.Schaden und E. Sim) 
Die operative Versorgung der Seitenbandrupturen am Daumengrundgelenk

(M. Cebulla, P. Konold, K. Frederking, E. Wemicke und A. Pannike) 436

Dynamische Zirkelextension zur Behandlung intraarticulärer Fingerfrakturen

(L.P.S.Stassen und Chr. van der Werken)

Konservative Behandlung veralteter Kapselverletzungen der

Fingermittelgelenke (A. Stock und B. Schimpfle)

Mechanik des Fingermittelgelenkes - Videoanalyse und Computersimulation

(T. Gaudernak, W. Hintringer, M.Leixnering und B.Schmiedmayer)

Muskelphysiologie, Sportverletzungen, funktionelle Therapie

Musculäre Gelenkstabilisierung nach Kniebandoperationen

in neuer achsloser Knieführungsschiene (J.Dippold, M. Martin und K. Börnert) ...

Behandlung der geschlossenen Unterschenkelfraktur:

Bedeutung des von E. Rehn beschriebenen „Muskelstupors“.

Elektromyographische Untersuchungen (S. Grafe)

Experimentelle Untersuchungen zur musculären Stabilisierung

des Kniegelenkes (J.Dippold und K. Börnert)

Chronische Knieinstabilitäten und „Sportfähigkeit“.

Studie bei 582 aktiven Fußball- und Handballspielern

(J.Pöhlmann, T. Werlich, H. Brand und V. Echtermeyer)

Mediale Kapsel-Band-Ruptur des Sportlerknies - genügt die funktionelle Therapie den hohen Ansprüchen? (A. Ekkernkamp, J. Brand und K. Neumann)

Langzeitergebnisse nach konservativer Therapie der isolierten vorderen

Kreuzbandruptur (K.P. Benedetto, Ch. Fink, Ch. Hoser und W. Glötzer)

Operative und konservative Therapie der Außenbandrupturen

am oberen Sprunggelenk - 10-Jahres-Spätergebnisse

(K. Neumann, W. Knopp und G. Muhr)

Funktionelle Behandlung und Nachbehandlung der ligamentären Instabilität am oberen Sprunggelenk (E.-A. Cramer und K. Friedhoff)

Stütz- und Bewegungssystem: Wirbelsäule, Hüftgelenk, Oberschenkel

Konservative und operative Behandlung der WS-Verletzungen am Brust-Lenden-Übergang (S. K. Erol, E. Serin, H. Havitçioglu, A. Ekin, H. Tatari und T.Kabaklioğlu)

Kritische Wertung der Ergebnisse des Fixateur interne zur operativen Behandlung instabiler Frakturen der Brust- und Lendenwirbelsäule (K. M. Stürmer, J. Hanke, H. Wissing, E. Nau, M. Schax und K. Koeser) 
Vorstellung eines neuen Fixatuer interne-Systems für die thoracolumbale

Wirbclsäule (D. Schulte-Bockholt, D. Puplat und L. Gotzen)

Darf die Umstellungsosteotomie und die Hüftkopfstanzung

bci fortgeschrittener Hüftkopfnekrose empfohlen werden?

(W. Stcinleitner, K. Rossak und M. Herzberger)

Myokardiale Ischämien beim Hüftgelenksersatz

(K. M. Peters, P.Peters, B.Schwanitz und K. W.Zilkens)

Biomechanische Untersuchungen zur Darstellung von Relativbewegungen verschiedener Prothesenschäfte im Implantatlager

(A. Bettermann, H. Ecke und M. Nietert)

Pauwels-III-Fraktur des Schenkelhalses - stabile Versorgung und Heilung ohne Osteotomie (A. Voorhoeve)

Chirurgische Behandlung der hüftnahen Femurfrakturen bei älteren Patienten

(S. K. Erol, H. Havitçioglu, A. Ekin, H. Tatari und T. Kabaklioğlu)

Erfahrungen mit einem verlängerten Gamma-Nagel für Problemfrakturen

des proximalen Femurs (J. W. J. L. Stapert, P. A. M. Vierhout und H. A. Schuppers) .

Stütz- und Bewegungssystem: Unterschenkel, Fuß

Neues 9mm-Marknagelsystem für Femur, Tibia und Humerus

(J.W. J. L. Stapert, P. A. M. Vierhout und H. A. Schuppers)

Korrekturosteotomien an Femur- und Tibiaschaft mit dem Verriegelungsnagel

(R. Kreusch-Brinker und G. Schwetlick)

Die kontinuierliche Korrektur von pseudarthrosebedingten

Extremitätenfehlstellungen mittels Fixateur externe (H.P. Kaps und J.Pfeil) ..... 455

Callusdistraktion an replantierten Unterschenkeln

(G. Giebel, C. Braun und O. Trentz)

Fibularesektion versus Fibulastabilisierung - Experimentelle Untersuchungen und klinische Praxis (W. Otto, K. Bartnig, H.-D. Pauer und Chr. Bierögel)

Indikation zur Anwendung der Syndesmosenplatte:

Biomechanische Grundlagen und klinische Langzeiterfahrungen

(J. Rödig, A. Leitner, A. Meißner und R. Rahmanzadeh)

Analyse klinischer und ganganalytischer Untersuchungen operativ und konservativ behandelter Patienten mit Luxationen im Bereich des Sprunggelenkes

(J. Singer, L. Brückner und K.-S. Pieper)

Komplikationen der offenen Fersenbeinosteosynthese

(R. Kadletz, K. P. Benedetto und B. Huber) 
Stütz- und Bewegungssystem: Pathophysiologie und Pathomorphologie

der Band- und Sehnenverletzungen

Trainingsinduzierte Sehnenhypertrophie am Schultergelenk beim Body-Building

(J.Jerosch, A. Ritchen und M. Marquard)

Strukturelle Altersveränderungen der „Rotatorenmanschette“

(M. Neurath, E. Stofft, F. Neurath und K. Neumann)

Ruptur der proximalen, langen Bicepssehne - Welche Tenodesetechnik

ist empfehlenswert? (R. Theermann, M. Krüger-Franke und H. J. Refior)

Ruptur der langen Bicepssehne. Biomechanisch begründete transhumerale Fixation

(M. Fritzsch und R. Labitzke)

Ruptur der distalen Bicepssehne: Indikation zur operativen Versorgung

(M. Krüger-Franke, R. Theermann und H. J.Refior)

Rekonstruktion des proximalen und distalen Kniegelenkstreckapparates

nach Patellaluxation: Ergebnisse (A. Sellmann, J. Petermann und L. Gotzen)

Rupturen des Kniestreckapparates: Spätergebnisse nach operativer Behandlung

(L. Rudig, J. Ahlers und K. Wenda)

Spätergebnisse isolierter und komplexer Bandverletzungen des Kniegelenkes unter Berücksichtigung der Arthrose und der Indikation zur Arthrodese

(R. Beickert, H. Brandner und J. Probst)

Morphologie der frischen vorderen Kreuzbandruptur im Rasterelektronenmikroskop

(S. Hom, K. Neumann und G. Muhr)

Der Kniebandschaden bei extraarticulären kniegelenknahen Frakturen

(M. Dickob und U. Mommsen)

Spätergebnisse isolierter und komplexer Bandverletzungen des Sprunggelenkes und besonderer Berücksichtigung der Arthrose und der Indikation zur Arthrodese (H. Hempfling, C.-U. Kütemeyer, K. Förster und J. Probst)

Technische Hilfen zur Funktionsdiagnostik, Orthesen

Knie-Arthrometer KT-1000: Stellenwert der instrumentellen Messung bei der Diagnose einer komplexen Knieinstabilität

(T. Werlich, J.Pöhlmann, H. Brand und V. Echtermeyer)

Neue Vorrichtung zur 3D-Untersuchung der Kniegelenksbewegung

(H. J. Hahne und H. W. Ulrich)

Stabilität verschiedener Orthesen bei definierter Knieinstabilität

(A. Schultz, T. Gaudernak, W. Schüller, H. Pelinka und A. Kerkoc)

Unterschenkel-Vacu-Cast: Unterdruck-Hülsenapparat als modellierfähiges, stabiles Gipsersatzsystem (P. Habermeyer) 
Funktionelle Behandlung mit der Aircast-Schiene oder Gipsruhigstellung bei der frischen fibularen Bandruptur des oberen Sprunggelenkes randomisierte klinische Studie (J. Klein, D. Rixen, B. M. Ure und Th. Tiling)

Knorpelschäden, Folgeeingriffe 478

Langzeitverläufe arthroskopisch verifizierter Knorpelläsionen

des Kniegelenkes (W. Jockers, M. Isay und W. Dick)

Die Behandlung der Osteochondrosis dissecans des Kniegelenkes durch die autologe Knorpel-Knochen-Transplantation (T. Wirth, G. Rauch, P. Schuler und P. Griss)

Osteochondrale Läsionen des oberen Sprunggelenkes.

Langzeitergebnisse nach operativer Behandlung

(J. Brand, A. Lies, A. Ekkernkamp und G. Muhr)

Distorsionstrauma des oberen Sprunggelenkes und Osteochondrosis Dissecans Tali:

Kann die Osteochondrosis Dissecans Tali traumatisch bedingt sein?

(J.Bruns und B. Rosenbach)

Gangbild nach Arthrodese des oberen Sprunggelenks.

Pedographische Untersuchungen (M.Zenkl, G. Bauer, R. Bensel,

T. Mittelmeier, H. Kurz und W. Mutschler)

Endoprothetik des oberen Sprunggelenkes. Indikation und Spätergebnisse

(B. Endrich und D. Terbrüggen)

Die Arthrodese mit cortico-spongiöser Spanplastik zum Talusersatz

am oberen und unteren Sprunggelenk (T. Schmickal und M. Rösgen)

Diskussion (H.Zwipp)

Pathophysiologie I: Mehrfachverletzungen, Begleitverletzungen, Blutverlust 486

Kombinationsverletzungen und Mortalität bei polytraumatisierten Patienten

(S. K. Erol, H. Tatari, H. Havitçioğlu, A. Ekin und T. Kabaklioğlu) 486

Messerstichverletzungen des linken Ventrikels im Rahmen eines komplexen

Suicidversuches (C. Schmid, J. Cremer, J. Sturm und J. Laas)

Pankreasverletzung durch Wirbelkörperbrüche des thoracolumbalen Überganges

(W. Koch, H. Messler, U.v. Deimling und B. Verhestraeten)

Spezielle diagnostische Aspekte abdominopelviner Läsionen bei instabiler

Beckenringverletzung (H. Rieger, D. Pennig, E. Brug, H. Bünte und W. Krings)

Die primäre interne Stabilisierung von dorsalen Beckenverletzungen

(S. B. Kessler, R.-W. Kenn, P. Krüger, H. Stützle und R. Frigg) 
XXX

Pathophysiologie II: Kompartment-Syndrom, Tourniquet-Syndrom,

Reperfusionsschaden

Das Kompartment-Syndrom - eine Komplikation der distalen Radiusfraktur?

Druckmessungen im Carpalkanal (Th. Peterson, K. Dresing, G. Schmidt

und K.P.Schmit-Neuerburg)

Dynamisches intrakompartmentales Druckverhalten in der

Tibialis-anterior-Loge bei maximaler Gehbelastung und beim Laufen

(J. Jerosch, S. Debus und B. Geske)

Tourniquet-induzierte Ischämie - Reperfusionsschäden beim Menschen

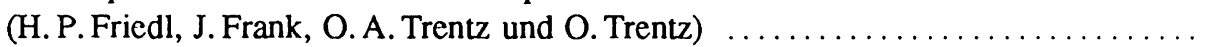

Diskussion (H.-D. Strube)

Knochenbank, Knochenersatz, biodegradable Materialien

Stabilität von humanem Knochen bei unterschiedlicher Konservierung und Sterilisation (J.Jerosch, H. Muchow und H.Clahsen)

Münchner Modell der allogenen Knochentransplantation

(G. O. Hofmann, M.F. Bauer, T. Wangemann, C. Falk, M.Zitzelsberger,

C. Hammer und G. Lob)

Knochenbanken in Deutschland - Ergebnisse einer Befragung und Konsequenzen

für den Anwender (J.Jerosch, M. Granrath und W. H. M. Castro)

Biologische Wirksamkeit einer dekalzifizierten

Humanknochen-Kollagen-Matrix (DBM): Klinische Studie (K. Riedel)

Resorbierbares Osteosynthese-Material: Indikationen, Vorgehen

und Ergebnisse nach klinischer Anwendung (R. Carbon, W. Link und H. Beck)

Wiederherstellung des frakturierten Radiusköpfchens mit resorbierbaren Stiften

(H. H. Schauwecker, B. Dreithaler und M. Kaiser) . . . . . . . . . . . . . . . . 499

Bandersatz am oberen Sprunggelenk mit Polydioxanon:

Experimentelle und klinische Untersuchungen (B.-W. Bär und W. Tausch) ...... 500

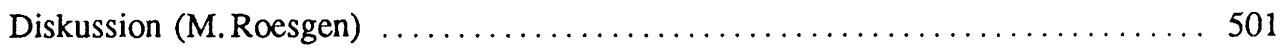

XI. Forum Experimentelle Unfallchirurgie $\ldots \ldots \ldots \ldots \ldots \ldots \ldots \ldots \ldots \ldots \ldots \ldots \ldots \ldots \ldots$

A. Schock, Organversagen, Ischämie, Reperfusionsschäden ............... 503

Der posturaumatische Permeabilitätsschaden mit nachfolgendem

Multiorganversagen nach Trauma: Eine Folge der Xanthinoxidase-Aktivierung?

(M. Maghsudi, M. L. Nerlich, J. A. Sturm und H.P.Friedl) 
Die chronische Endotoxinämie am Schaf als Modell eines Multiorganversagens

(A. Seckamp, A. Dwenger, G. Regel und J.A. Sturm)

Aktivierung perizentraler hepatischer Makrophagen nach hämorrhagischem Schock

an der Ratte (V. Bühren, I. Marzi, B. Kiefer und O. Trentz)

Lungenfunktion nach Oberschenkelmarknagelung im Staubschen Schafmodell Einfluß durch hämorrhagischen Schock und Lungenkontusion?

(H.-C.Pape, G. Regel, A. Dwenger und J. A. Sturm)

Reduktion hepatischer Mikrozirkulationsstörungen im

hämorrhagischen Schock durch humane Superoxid-Dismutase (h-SOD)

(I. Marzi, R. Hower, V. Bühren und O. Trentz)

Ischämie und Reperfusion der Skelettmuskulatur: Beurteilung

des postischämischen Reperfusionsschadens nach intermittierender

und kontinuierlicher Ischämie (M. D. Menger, D. Steiner und K. Meßmer)

Reperfusionsschäden durch toxische Sauerstoffradikale

nach Tourniquet-induzierter Ischämie beim Menschen

(H. P. Friedl, J. Frank, O. A. Trentz, U. Bauch, G. O. Till und O. Trentz)

Thromboseprophylaxe bei Hüftprothesenoperationen durch Begrenzung der Knochenmarkeinschwemmungen (K. Wenda, G. Ritter, K. Hahn und J.K. Seifert)

B. Immunologie, Infekt, Sepsis

Vergleichende Analyse der postoperativen systemischen zellmediierten Immunität bei Patienten mit und ohne Fremdkörperimplantation (Th. Griga, Ch. Josten, R. Sistermann und G. Muhr)

Ergebnisse der ${ }^{99 m}$ Tc-Leukocyten-Szintigraphie bei der Diagnostik

der akuten und chronischen Infekte in der Traumatologie

(Th. Hupp, G.Schäffer, W. Friedl und W. Ruf)

Einfluß von Ibuprofen auf den Serumspiegel des Prostaglandins $E_{2}$

und des freien Interleukin 2-Receptors bei der Osteitis

(Ch. Josten, Th. Griga und G. Muhr)

Gestörte hepato-celluläre Calcium-Regulation während gram-negativer Sepsis

in der Ratte (S. Rose, H. Wünstel, O. Trentz und M. M. Sayeed)

Betalactam-induzierte Serumresistenzreduktion bei aus Blutkulturen

von Sepsisfällen isolierten Escherichia coli-Stämmen

(B. Meyer-Berendes, H. Leying, W. Opferkuch und G. Muhr)

Makrophagendefekte bei posttraumatischer Osteitis

(K. M. Peters, G.Zwadlow-Klarwasser, K. Koberg und K. W.Zilkens)

Diskussionen (A.Seekamp) 
Mikrocallusformationen - Chronische Traumata des Skelettsystems als Stimulus des physiologischen Knochenumbaus?

(M. Hahn, M. Vogel, H. U. Langendorff und G. Delling)

Der Einfluß extracorporaler Stoßwellen auf die Knochenbruchheilung

(G. Haupt, A. Ekkernkamp, M. Chvapil, A. Haupt und B. Gerety)

Primäre und sekundäre Frakturheilung - Ein Widerspruch?

(Th. Rack und K. M. Stürmer)

Dreidimensionale Messung von Fragmentbewegungen am Frakturspalt -

Biomechanische Studie an experimentellen Tibiafrakturen

(R. Hoffmann, H. McKellop, A. Sarmiento und B.Lu)

Callotasis: Ein Verfahren zur biologisch-statistischen Bewertung

der knöchernen Heilung (R. Schlenzka, M. Stamm und C. Pistor)

Der Einfluß von Stabilität und Vascularität auf die ossäre

Regeneration einer Mehrfragmentfraktur (U. Heitemeyer)

Überbrückung von Knochensegmentdefekten mit autogen vascularisierten

allogenen Knochen - Tierexperimentelle Studie und klinische Perspektive

(C. Braun und M. Bauer)

Stellenwert von Muskellappen bei avasculärem Knochen

(R. Ketterl, R. Ascherl, H. U. Steinau und B. Claudi)

Morphologie autologer und homologer Rippenknorpeltransplantate

in Epiphysenfugendefekten beim Göttinger Minipig

(M. Dallek, N. Meenen und K.-H. Jungbluth)

Morphometrische Gefäßbaumanalysen der distalen Unterarmepiphysen

nach experimenteller Fraktur und operativer Versorgung

(G. Benz, G. Mall und R. Daum)

D. Ionisierende Strahlen, bildgebende Verfahren

Histologische Auswirkungen der Strahlentherapie nach Plattenosteosynthese am Kaninchenfemur (H. G. Hermichen, Th. Kaulich, B. Schmidt und C.-P. Adler) . . 534

Die Darstellung der traumatisierten Bandscheibe durch Kernspintomographie und intraoperative Discographie (S. v. Gumppenberg, B. Allgayer, J. Vieweg und B. Claudi)

3-D-Rekonstruktion knöcherner Verletzungen am Unfalltag Korrelation zwischen Röntgenbild und 3. Dimension

(J. V. Wening, K. H. Jungbluth, B. Fink und B.Pflesser)

Grauwert-Bildananlyse von Mikroradiographie-Präparaten

(K. Wolf, M. Puhlmann, W. Stock und S. Kessler) 
Ist die Sonographie nach Spongiosatransplantation eine Alternative Ergebnisse einer tierexperimentellen Studie

(H. B. Reith, W. Harmann und W. Kozuscheck)

Sterilisation und Kryokonservierung von Bankknochen?

Biomechanische Untersuchungen

(G. Voggenreiter, R. Ascherl, M. A. Scherer, H. J. Früh, H. Knaepler

und G. Blümel)

Desinfektion allogener Spongiosatransplantate durch thermisch-homogene

Behandlung im Hochfrequenzfeld (H.-M.Seipp und H. Knaepler)

Thermische Desinfektion allogener Spongiosatransplantate

im Inkubationsverfahren bei $80^{\circ} \mathrm{C}$

(H.-M. Seipp, H. Knaepler, B. Dreilich und T. von Garrel)

Experimentelle Untersuchung zur Antigenität von sterilisierten

Knochentransplantaten (H.-E. Schratt, J. L. Spyra, G. Voggenreiter,

R. Hipp, J. Tübel und G. Blümel)

Die Einheilungsdynamik sterilisierten Knochens - Tierexperimentelle

Untersuchungen (H. Knaepler, D. Sand, H. Rath und T. v. Garrel)

Zur Induktion periostaler Knochenneubildung durch osteoperiostale Expansion:

Tierexperimentelle Ergebnisse (M. Cornils, R. Schanz und L. Meiss)

Knochenneubildung im ersatzschwachen Lager - Tierexperimentelle

Untersuchungen zur Kombination von Knochenersatzstoff und freien

Periosttransplantaten (W. Klaes, St. Assenmacher, K. M. Stürmer

und K.-P. Schmit-Neuerburg)

Das Degradationsverhalten der Calciumphosphatkeramiken Hydroxylapatit

und Tricalciumphosphat im Verlauf der knöchernen Integration

(M. Roesgen und G. Hierholzer)

Knochenneubildung im langstreckigen Tibiaschaftdefekt nach Implantation demineralisierter Knochenmatrix (H. Stützle, S. Kessler, K. Hallfeldt und L. Schweiberer)

Defektüberbrückung mit Hydroxylapatit an der Femurdiaphyse der Ratte (P. Behrens, C. Alfke, H.-J.Egbers, B. Simons, E. Striepling und W.Zenker) . . ... 550

Diskussion (V.Studtmann)

Experimentelle Untersuchungen zur Belastung der Symphyse beim „Gehen“ und Vergleich von Symphysenstabilisierungsverfahren unter simulierten dynamischen Belastungen (A. Meißner, R. Wilk und R. Rahmanzadeh) 
Rigide oder dynamische Stabilisierung der Symphyse bei ligamentärer

Beckenringinstabilität? (J. Szita, H.E. Bär und G. Muhr)

Biomechanische Untersuchungen zur Beanspruchung und Stabilität

des oberen Sprunggelenkes (S. Rübenacker, L. Claes, P. Becker und H. Gerngroß) . 555

Glykosaminoglykane in der Bandheilung - Eine tierexperimentelle Studie

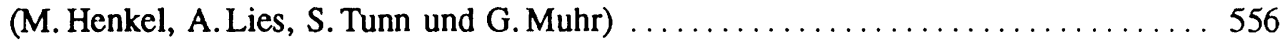

Physiodegeneration der proximalen, langen Bicepssehne - Wo ist der

Locus minoris resistentiae? (R. Theermann, H.J. Refior und A. Kaltenecker)

Steigerung der Sehnenreißfestigkeit unter Einwirkung eines Huminates (HS 1500)

(W. Schlickewei, U.N. Riede und E.H.Kuner) $\ldots \ldots \ldots \ldots \ldots \ldots \ldots \ldots \ldots$

Zugfestigkeit tiefgefrorener und lyophilisierter humaner

Achillessehnen nach Gamma- und Ethylenoxid-Sterilisation

(G. Rauch, M. Gerbersdorf, P.Dörner und P. Griss)

Quantitative rasterelektronenmikroskopische Untersuchungen

der spontan heilenden Achillessehnentenotomie beim Schaf

(K. Günther, H.-P. Scharf und W.Puhl)

G. Kniegelenk: Plastik und Ersatz der Kreuzbänder

Biomechanische Untersuchungen zum Effekt lateraler Stabilisierungen

auf die Spannung des vorderen Kreuzbandes

(P. Lobenhoffer, C. Krettek, T. Gerich und N. Haas)

Die Bedeutung der Vorspannung bei gestielten Patellarsehnentransplantaten

zur Rekonstruktion des vorderen Kreuzbandes (M. A. Scherer, R. Ascherl,

T. Brunner, H. J. Früh, W. Erhardt und G. Blümel)

Wassergehalt als Parameter der Ligamentisation nach autoplastischem

Kreuzbandersatz? (M. A. Scherer, R. Ascherl, W. Siebels, K. Lehner,

R. Gradinger und G. Blümel)

Mikrovasculäre und immunhistologische Ergebnisse homolog

transplantierter vorderer Kreuzbänder am Kaninchenmodell

(B. Fromm, B. Krause und H. Cotta)

Tierexperimentelle Untersuchung lösungsmittelkonservierter

Tibialis-anterior-Sehnen als bindegewebiges Transplantat für den Ersatz

des vorderen Kreuzbandes (H.-P.Scharf, H.-J.Pesch und W.Puhl)

Zur Problematik des alloplastischen Ersatzes des vorderen Kreuzbandes -

Experimentelle Ergebnisse (R. Ascherl, W. Siebels, M. A. Scherer, R. Gradinger,

E. Hipp und G. Blümel)

Veränderungen der extracellulären Matrix eines freien

Patellarsehnentransplantates beim hinteren Kreuzbandersatz

(U. Bosch, A. Nerlich, B. Decker, W. Kasperczyk und H.J. Oestern) 
Untersuchungen zur Isometrie des hinteren Kreuzbandersatzes

(J. Petermann, P. Trus und L. Gotzen) $\ldots \ldots \ldots \ldots \ldots \ldots \ldots \ldots \ldots \ldots \ldots \ldots$

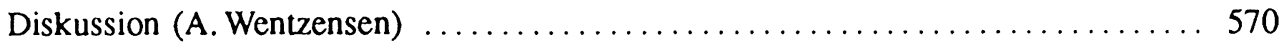

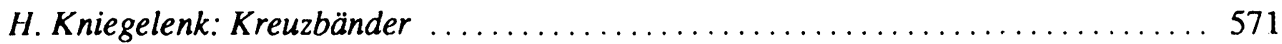

Funktion und Längenänderung der Faserbündel der Kreuzbänder

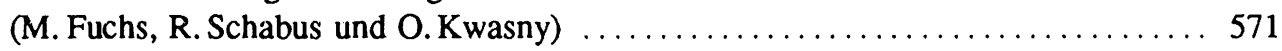

Experimentelle Untersuchung zum Einfluß von Muskelzug und äußeren Kräften auf das Dehnungsverhalten der menschlichen Kniebänder

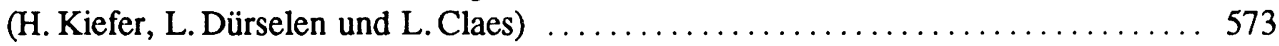

Computersimulation der Kreuzbandfunktion - Einfluß der Fixationspunkte auf das Bewegungsausmaß und die Stabilität

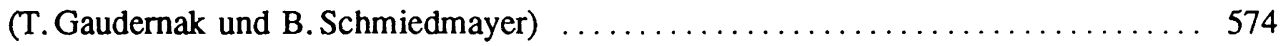

Einfluß des Testprocedere auf die Ergebnisse biomechanischer

Belastungstests an Kniebändern (W.J. Kasperczyk, U. Bosch, L. Borchers,

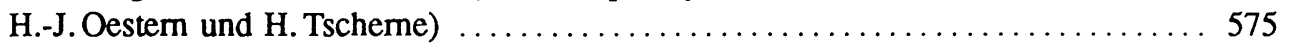

Synoviale Fremdkörperreaktion im Kniegelenk des Schafes beim Ersatz des vorderen Kreuzbandes durch ein Kevlarband

(C. Tesch, J. V. Wening, I. Cordes und H.-U. Langendorff) 576

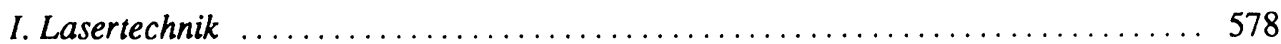

Athermische Laser und ihre Bedeutung für die Unfallchirurgie

(W.Neu, M. Dressel, R. Jahn, K.F. Klein, H. U. Langendorff und K. H. Jungbluth) . . 578

Schneiden und Bohren von Knorpel- und Knochengewebe mit Excimerlasem

(R. Jahn, M. Dressel, H. U. Langendorff, W. Neu und K. H. Jungbluth) . . ...... 579

$\mathrm{CO}_{2}$-Laser-Einsatz in der rekonstruktiven Gefäßchirurgie -

Tierexperimentelle Studie (A. Ahmadi, M. Böhm und T. Mühlberger) . . . ..... 580

Die laserunterstützte Mikroanastomose - Eine experimentelle Untersuchung

(W. Knopp, G. Dasbach, W. Marek, B. Voss, G. Muhr und K.-M. Müller) . . ...... 581

Einsatz des $\mathrm{CO}_{2}$-Lasers bei der Entfernung zementierter Hüftgelenksendoprothesen

(R. Inglis, A. Hermanni, J. Windolf und A. Pannike) $\ldots \ldots \ldots \ldots \ldots \ldots \ldots \ldots \ldots 2$

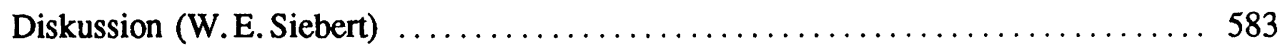

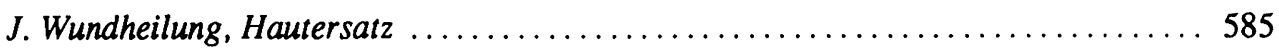

Insulin-like Growth Factors (IGF) I und II und IGF-bindendes Protein 3 (IGF-BP-3) bei polytraumatisierten Patienten (E. Stöhr, W. Blum, M. Ranke und $S$. Weller) 
Ein neues Modell zur Untersuchung der Wundheilung im ischämischen Gewebe. Beschleunigung der Wundheilung durch Buflomedil (M. Kamler, R. K. Saetzler, H. A. Lehr, T. J. Galla und K. Messmer)

Morphologische und histochemische Untersuchungen der Wundoberfläche bei der Verwendung von Hautersatzmaterialien (K. Weise, Ch. Klessen und A. Manger)

Vergleichende experimentelle Stabilitätsuntersuchungen

zu Osteosyntheseverfahren bei Densfrakturen (H.-J. Wilke, K. Fischer,

A. Kugler, F. Magerl, O. Wörsdörfer und L. Claes) $\ldots \ldots \ldots \ldots \ldots \ldots \ldots \ldots$

Biomechanische Analyse lumbaler Wirbelkompressionsfrakturen

(R. Steffen, L.-P. Nolte, E. Schopphoff und J. Krämer)

Stabilität des distalen Radioulnargelenkes bei Fraktur des Radiusschaftes

(Galeazzi-Frakturen) (O. Kwasny, M. Fuchs und H. Hertz)

Überlegungen zur Rekonstruktionierbarkeit des Discus articularis

des distalen Radioulnargelenkes (Spannungsverhalten und Durchblutung)

(O. Kwasny, M. Fuchs und R. Weinstabl)

Die mediale Schenkelhalsfraktur - Eine histomorphologische Strukturanalyse

zur Aufdeckung neuer Gesichtspunkte in der Pathogenese der Erkrankung

(M. Vogel, H.-U. Langendorff, M. Hahn und G. Delling)

Das Frakturmodell einer subtrochanteren Mehrfragmentfraktur am Schaf -

Entwicklung und Anwendung (F. Baumgaertel, B. Rahn und S. M. Perren)

Druck- und Kontaktflächenänderung am Talus nach Calcaneustrümmerfraktur

(O. Paar, R. Kasperk und S. Eren)

Der Einfluß verschiedener Titanoberflächen auf die Scherfestigkeit

an der Grenzfläche zwischen Implantaten und Knochen

(L. Claes, H.-J. Wilke und S. Steinemann)

Biomechanik und Knochenheilung bei einer „No Contact Plate“

(Plattenfixateur) (B. Hartung, R. Henke und U. Fuhrmann)

Tierexperimentelle Untersuchungen über die Haftfestigkeit verschiedener

Oberflächenbeschichtungen am Knochen (A. David, A. Pommer, J. Eitenmüller

und G. Muhr)

Polydioxanon als Bandscheibenersatz - Ergebnisse einer

tierexperimentellen Studie (B. Rischke, K. Westermann und M. Samii) 
Vergleichende tierexperimentelle Untersuchung zum knöchernen Einwachsverhalten von HA-beschichteten Reintitangitternetzen im infizierten Milieu (A. Wilke, J. Orth, M. Kraft und P. Griss) 602

Der Einfluß einer bakteriellen Kontamination auf die Degradation von biodegradierbaren Implantaten (G. O. Hofmann, H. Liedtke, G. Ruckdeschl und G. Lob) 603

Gestaltoptimierung von Osteosyntheseschrauben (Th. Mittlmeier, C. Mattheck, A. Baumgartner und G.Lob) 604

Zugfestigkeit, Drehmoment und Ausreißkräfte selbstschneidender und herkömmlicher Corticalisschrauben (Th. Hess, Th. Hopf, E. Fritsch und H. Mittelmeier)

Der Einfluß der Knochendichte und des Schraubendesigns auf die Verankerung von Pendikelschrauben (R. H. Wittenberg, M. S. Coffee, J. Grifka, K. S. Lee,

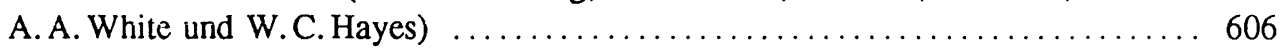

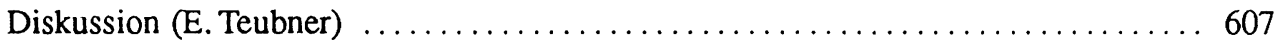

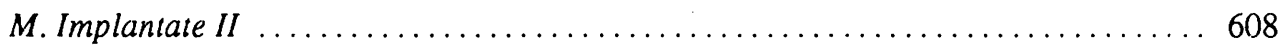

Physikalische Auswirkungen des Einschlagvorgangs bei der Marknagelung des Femur (W.-D.v. Issendorff, G. Ritter, J. Ahlers und K. Wenda) . . . . . . . . . . 608

Biomechanische Untersuchungen zu einem neuen Krallen-Verriegelungsnagel (KVN) für Femurschaftfrakturen (C. Krettek, W. Mengert, N. Haas, R. Mathys sen. und H. Tscherne) 609

Biomechanik des neuen, nicht aufgebohrten massiven AO-Unterschenkelverriegelungsnagels und des konventionellen Universalverriegelungsnagels im Vergleich (P.Schandelmaier, C. Krettek, N. Haas und H. Tscherne)

Die Anwendung des Fixateur interne bei Frakturen langer Röhrenknochen im Tierexperiment (R. Seibold, A. Betz, L. Schweiberer und S. Perren)

Ergebnisse nach Hemiarthroplastik des Hüftgelenkes mit Hydroxylapatit beschichteter Titanprothese - Tierexperimentelle Untersuchung (J. Orth, P. Griss, J. Falkenburg und H. Kienapfel)

Mechanische Untersuchung eines neuen flexiblen Prothesenschaftes für das Femur (G. Zeiler) 614

Diskussion (D. Höntzsch) 615 
Einleitung zum Thema (J. Probst)

Zweckbestimmung des Gutachtens (J.Probst)

Vorbereitung des Gutachtens (J.Probst und G. Hofmann) 620

Formeller Aufbau des Gutachtens (M. H. Ruidisch)

Akten und Fremdanamnese (M. Graeber) 626

Klinische gutachterliche Befunderhebung (M.H.Ruidisch) 628

Die manualmedizinische Befundherhebung an der Wirbelsäule unter gutachterlichen Gesichtspunkten (W. Treioel und Th. Laser)

Ergänzende Untersuchungen: Konventionelle Röntgenuntersuchung, CT, MR, Szintigraphie, Labor (H. E. Mentzel)

\section{B. Gutachterliche Beurteilung der verletzten Wirbelsäule}

Anspruchsgrundlagen GUV, PUV, Haftpflichtschaden (J. Probst)

Gutachterliche Abgrenzung unfallfremder Befunde (H. Bilow)

Einteilung der Wirbelsäulenverletzungen unter gutachterlichen

Gesichtspunkten (M. H. Ruidisch)

Analyse und Bewertung des Verletzungsmechanismus (H.E. Mentzel)

Stellung und Zweck der krankengymnastischen Behandlung beim Unfallverletzten (E. Borlinghaus)

Stellung und Zweck der Balneotherapie und Massage beim Unfallverletzten (M. Graeber)

Stellung und Zweck der Ergotherapie beim Unfallverletzten

Indikation zur Krankengymnastik in der operativen und konservativen

Frakturbehandlung (M. Gutbier)

Indikation zur Krankengymnastik in der postoperativen Behandlung von Gelenkbandverletzungen (A. Wentzensen) 
Allgemeine Grundlagen der Indikation stabilisierender und mobilisierender Heil- und Hilfsmittel und versicherungsrechtliche Aspekte

(R.-A. Grünther und O. Oest)

Indikation zu Heil-/Hilfsmitteln nach Wirbelsäulenverletzungen

(M. H. Ruidisch)

Indikation zu stabilisierenden Heil- und Hilfsmitteln nach Verletzungen

an den oberen Gliedmaßen (D. Lazovic)

Indikation zu mobilisierenden Heil- und Hilfsmitteln nach Verletzungen an den oberen Gliedmaßen (F. Gossé)

XIII. Wissenschaftliche Ausstellung, Posterausstellung

Experimentelle Untersuchungen zur Wirkung von Lidocain beim Hirnödem

(R. Ascherl, M. Schimmer, A. Müller, M. Schuback und G. Blümel)

Tierexperimentelle Untersuchungen zur Knochenneubildung durch freie

Periosttransplantate (St. Assenmacher, W. Klaes, K. M. Stürmer

und K.-P.Schmit-Neuerburg)

Laser als Ersatz für Säge und Schere in der Unfallchirurgie?

Eine vergleichende experimentelle Studie (M. Dressel, R. Jahn,

H. U. Langendorff, W. Neu und K. H. Jungbluth)

Aufbau und Funktion eines Lehr- und Übungsmodells für die Versorgung

von Schädel-Him-Verletzten (G. Feuchtgruber, K. Geissler, K.-G. Kanz

und L. Schweiberer)

Biomechanische Eigenschaften von bovinen, chemisch konservierten Schrauben

aus Knochenmaterial (H. J. Früh, R. Ascherl, M. A. Scherer und G. Blümel)

Resorbierbare Schrauben aus Polyglykolid: Erste klinische Erfahrungen -

Möglichkciten, Grenzen (H. Gerngroß, M. A. Scherer und R. Steinmann)

Digitales mobiles Kompartmentdruck-Meßsystem

(H. Gerngroß und M. N. Rosenheimer)

Zur Einheilung von ganzbeschichteten, zementlosen Schaftprothesen

am Beispiel der Metallspongiosa - Experimentelle Untersuchungen

(R. Gradinger, S. Wicke-Wittenius, R. Ascherl, M.-L. Schmeller,

W. Erhardt, W.Plötz und E. Hipp)

Zum Problem der heterotopen Ossifikationen - Histologische Untersuchungen

(C. Hegerl, R. Ascherl, R. Hipp, B. Stübinger und G. Blümel)

Interdisziplinäre onkologische, urologische und plastisch-chirurgische Behandlung der Condylomata Acuminata Gigantissima (Buschke-Löwenstein Tumor) (R. Inglis, W. Meyer, J. Windolf, W. Boeckmann, A. Pannike und D. Jonas) 
Systematik der Einteilung des traumatischen Weichteilschadens analog zur AO-Klassifikation der Frakturen (R. Inglis, J. Rueger, J. Windolf und A. Pannike) . . 694

Ultrastrukturelle Untersuchung des Trevirabandeinbaus nach vorderem Kreuzbandersatz (H.-J. Koch, K. M. Stürmer und R. Letsch) 696

Polytraumascores - Verletzungen und Parameter im Vergleich

(R. Ascherl, M.Leonardi, M. A. Scherer und G. Blümel)

Vergleich verschiedener Stabilisierungsverfahren für gerissene Symphysen im selbstentwickelten Simulator für Gangbedingungen

(A. Meißner, R. Wilk und U. Boenick) 698

Resorbierbares PDS-Fixationsmaterial versus Drahtcerclage biomechanische Untersuchung zur Schultereckgelenkstabilisierung (M. Sangmeister, H. Windhagen und L. Gotzen)

Experimentelle Untersuchungen zur Primärnaht der isolierten Kreuzbandruptur (M. A. Scherer, R. Ascherl, H.J. Früh, R. Gradinger, E. Hipp und G. Blümel) 700

Dokumentation im Notarztwagen mit einem Protokoll (J. Windolf, M. Dickopf, R. Inglis und A. Pannike) 701

XIV. Wissenschaftliche Filme/Video 703

Das Kompressionssyndrom des N. radialis am Ellenbogen

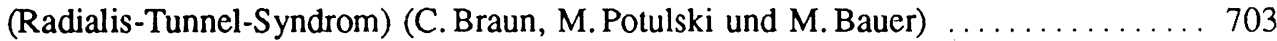

Kompartment-Syndrom - Manuelle und digitale Druckmessung (V.Echtermeyer) . 704

Möglichkeiten der sonographischen Diagnostik von Knieband- und

Meniscusverletzungen (W. Friedl, U. Göhring und St. Post) 705

Belastungsstabile Versorgung von Problemverlctzungen des proximalen Femurendes (W. Friedl und St.Post) 706

Komplikationen und ihre operative Behandlung nach primär konservativ versorgter frontobasaler Verletzung - Fallstudie mit Langzeitverlauf (P. Knöringer)

Die Extensor Indicis Plastik (Videofilm) (M.Leixnering und W. Hintringer) 707

Traumamanagement Teil I (Lehrfilm $16 \mathrm{~min}$ )

Organisation und Ablauf der medizinischen und technischen Hilfe bei der Unfallverletzung (U. Malewski, K. Hette, A.Zielke, F. Wranze

und L. Gotzen 709

Schulter-Arthroskopie nach frischer Luxation - Ein Wegweiser für das therapeutische Procedere (N. M. Meenen, J. V. Wening, K. H.Jungbluth und H. Schöntag) 
Supinatorschlitzsyndrom und Subluxation des Radiusköpfchens nach alter Fraktur im Jugendalter - Implantation eines Silastic Radial Heads sowie Dekomprimierung im Supinatorschlitz (A. Obiltschnig und D. Szolar)

Die biologische Wirksamkeit des fasergeführten Excimerlaserstrahls auf verschiedene organische Gewebe (R. Jahn, M. Dressel, H.U.Langendorff,

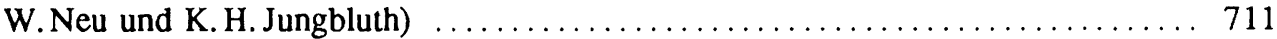

Behandlung der chronischen Osteitis (R. Neugebauer) $\ldots \ldots \ldots \ldots \ldots \ldots \ldots \ldots 71$

10-Jahresergebnisse nach Innenmeniscus-Korbhenkelresektion unter arthroskopischer Sicht (F. Farid)

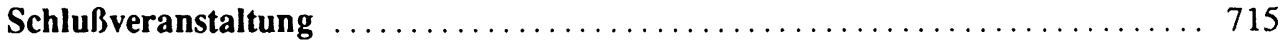

Freie Berufe in einer freiheitlichen Ordnung (W. Hamm) $\ldots \ldots \ldots \ldots \ldots \ldots \ldots \ldots$

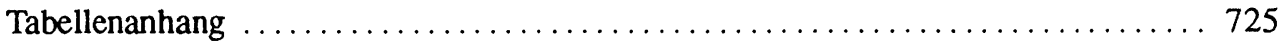

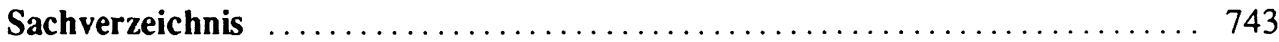




\title{
Die prognostische Aussagekraft biochemischer Parameter im Vergleich zum ISS und PTS beim Polytrauma
}

\author{
D. Nast-Kolb, Ch. Waydhas, M. Jochum und L. Schweiberer
}

Chirurgische Klinik der Innenstadtklinik, Universität München, Nußbaumstraße 20, W-8000 München2, Bundesrepublik Deutschland

In einer prospektiven Studie wurden 100 polytraumatisierte Patienten (mittl. ISS: 37, mittl. PTS 38 Punkte) ab Klinikaufnahme über einen 14-tägigen Beobachtungszeitraum untersucht. 16 Patienten verstarben sekundär im Multiorganversagen (4.-28. Tag), 47 Patienten überlebten mit und 37 ohne definierte Organfunktionsstörungen.

Bei Klinikaufnahme unterschieden die Serinproteinase PMN-Elastase (ng/ml), die Cysteinproteinase Kathepsin B (mU/l) sowie die AT III-Konzentration (\%) signifikant (p $<0,01)$ zwischen Patienten mit und ohne späterem Organversagen. Die später Versterbenden wurden zu diesem Zeitpunkt durch die Serumlaktatspiegel (mg/dl), am 4. Tag auch durch die PMN-Elastase $(\mathrm{ng} / \mathrm{ml}), \mathrm{CRP}(\mathrm{mg} / \mathrm{dl})$ sowie Neopterin $(\mathrm{mmol} / \mathrm{l})$ von den Überlebenden signifikant $(p<0,01)$ abgegrenzt. Zur Überprüfung der prognostischen Relevanz dieser Mediatoren wurden Sensitivität, Spezifität, positiv und negativ prädiktiver Wert im Vergleich zu den Traumascores ISS und PTS ermittelt (Tabelle 1, 2).

Bezüglich der Vorhersage des Organversagens ergaben die Traumascores sowie die biochemischen Faktoren mit 62-77\% richtigen Aussagen ein annähernd gleiches Ergebnis, wobei der PTS tendenziell am besten abschnitt. Bezüglich späteren Versterbens zeigten die Laborparameter mit 84-90 \% richtigen Vorhersagen eine größere Genauigkeit. Die Kombination dieser Mediatoren ließ die Aussagekraft weiter verbessern: Waren mindestens 3 oder

Tabelle 1. Vorhersage von Organversagen bei Klinikaufnahme

\begin{tabular}{llllll}
\hline Diskriminanzwerte & $\begin{array}{l}\text { ISS } \\
\text { (Pkt.) }\end{array}$ & $\begin{array}{l}\text { PTS } \\
>30 \\
(\text { Pkt.) }\end{array}$ & $\begin{array}{l}\text { Elastase } \\
>200 \\
(\mathrm{ng} / \mathrm{ml})\end{array}$ & $\begin{array}{l}\text { Kathepsin B } \\
(\mathrm{mU} / \mathrm{1})\end{array}$ & $\begin{array}{l}\text { AT III } \\
<80 \% \\
(\%)\end{array}$ \\
\hline Sensitivität & 71 & 82 & 83 & 50 & 79 \\
Spezifität & 46 & 68 & 44 & 89 & 52 \\
Pos. präd. Wert & 69 & 81 & 73 & 90 & 74 \\
Neg.präd. Wert & 49 & 69 & 58 & 48 & 59 \\
\hline
\end{tabular}

Tabelle 2. Vorhersage von Versterben bei Klinikaufnahme* und am 4. Tag

\begin{tabular}{lllllll}
\hline Diskriminanzwerte & $\begin{array}{l}\text { ISS } \\
>40 \\
(\text { Pkt.) }\end{array}$ & $\begin{array}{l}\text { PTS } \\
>50 \\
(\text { Pkt.) }\end{array}$ & $\begin{array}{l}\text { Elastase } \\
>500 \\
(\mathrm{ng} / \mathrm{ml})\end{array}$ & $\begin{array}{l}\text { CRP } \\
>20 \\
(\mathrm{mg} / \mathrm{dl})\end{array}$ & $\begin{array}{l}\text { Neopterin } \\
(\mathrm{mmol} / \mathrm{l})\end{array}$ & $\begin{array}{l}\text { Laktat* } \\
\text { (mg/dl) }\end{array}$ \\
\hline Sensitivität & 60 & 47 & 56 & 57 & 77 & 60 \\
Spezifität & 67 & 86 & 91 & 95 & 80 & 89 \\
Pos.präd. Wert & 24 & 37 & 56 & 67 & 42 & 50 \\
Neg.präd. Wert & 90 & 90 & 91 & 93 & 95 & 92 \\
\hline
\end{tabular}


4 Faktoren pathologisch, so betrug die Letalität $89 \%$, waren alle 4 Faktoren unauffällig, so überlebten $98 \%$. Die Auswertungen zeigen, daß biochemische Meßparameter bei der Prognosebeurteilung den der subjektiven Einschätzung des Untersuchers unterworfenen Traumascores zumindest gleichwertig sind und somit als objektive Kriterien empfohlen werden können. 